\title{
De novo assembly and analysis of the transcriptome of the Dermacentor marginatus genes differentially expressed after blood-feeding and long-term starvation
}

\author{
Ercha Hu${ }^{1,2+}$, Yuan Meng ${ }^{3+}$, Ying $\mathrm{Ma}^{2}$, Ruiqi Song ${ }^{1,2}$, Zhengxiang $\mathrm{Hu}^{5}$, Min $\mathrm{Li}^{2}$, Yunwei Hao ${ }^{2}$, Xinli Fan², \\ Liting Wei ${ }^{2}$, Shilong Fan ${ }^{2}$, Songqin Chen ${ }^{2}$, Xuejie Zhai ${ }^{2}$, Yongchang $\mathrm{Li}^{1,4}$, Wei Zhang ${ }^{2}$, Yang Zhang ${ }^{2}$, \\ Qingyong $\mathrm{GuO}^{2^{*}}$ and Chahan Bayin²*
}

\begin{abstract}
Background: The ixodid tick Dermacentor marginatus is a vector of many pathogens wide spread in Eurasia. Studies of gene sequence on many tick species have greatly increased the information on tick protective antigen which might have the potential to function as effective vaccine candidates or drug targets for eco-friendly acaricide development. In the current study, RNA-seq was applied to identify D. marginatus sequences and analyze differentially expressed unigenes.
\end{abstract}

Methods: To obtain a broader picture of gene sequences and changes in expression level, RNA-seq was performed to obtain the whole-body transcriptome data of D. marginatus adult female ticks after engorgement and long-term starvation. Subsequently, the real-time quantitative PCR (RT-qPCR) was applied to validate the RNA-seq data.

Results: RNA-seq produced 30,251 unigenes, of which 32\% were annotated. Gene expression was compared among groups that differed by status as newly molted, starved and engorged female adult ticks. Nearly one third of the unigenes in each group were differentially expressed compared to the other two groups, and the most numerous were genes encoding proteins involved in catalytic and binding activities and apoptosis. Selected up-regulated differentially expressed genes in each group were associated to protein, lipids, carbohydrate and chitin metabolism. Bloodfeeding and long-term starvation also caused genes differentially expressed in the defense response and antioxidant response. RT-qPCR results indicated 6 differentially expressed transcripts showed similar trends in expression changes with RNA-seq results confirming that the gene expression profiles in transcriptome data is in consistent with RT-qPCR validation.

Conclusions: Obtaining the sequence information of D. marginatus and characterizing the expression pattern of the genes involved in blood-feeding and during starvation would be helpful in understanding molecular physiology of $D$. marginatus and provides data for anti-tick vaccine and drug development for controlling the tick.

Keywords: Dermacentor marginatus, RNA-seq, Transcriptomics, Tick physiology, Differential expression

*Correspondence: 83949588@qq.com; 2514062881@qq.com

†Ercha Hu and Yuan Meng contributed equally to this work

${ }^{2}$ College of Veterinary Medicine, Xinjiang Agricultural University,

Ürümqi 830052, Xinjiang Uygur Autonomous Region, People's Republic of China

Full list of author information is available at the end of the article

\section{Background}

Ticks are obligate hematophagous ectoparasites of great medical and veterinary importance [1]. Dermacentor marginatus Sulzer that is a wide spread tick species of

c) The Author(s) 2020. This article is licensed under a Creative Commons Attribution 4.0 International License, which permits use, sharing, adaptation, distribution and reproduction in any medium or format, as long as you give appropriate credit to the original author(s) and the source, provide a link to the Creative Commons licence, and indicate if changes were made. The images or other third party material in this article are included in the article's Creative Commons licence, unless indicated otherwise in a credit line to the material. If material is not included in the article's Creative Commons licence and your intended use is not permitted by statutory regulation or exceeds the permitted use, you will need to obtain permission directly from the copyright holder. To view a copy of this licence, visit http://creativecommons.org/licenses/by/4.0/. The Creative Commons Public Domain Dedication waiver (http://creativecommons.org/publicdomain/zero/1.0/) applies to the data made available in this article, unless otherwise stated in a credit line to the data. 
the Ixodidae carries and transmits a variety of tick-borne pathogens to livestock and human, such as Babesia [2], Theileria [3], Anaplasma [4], Rickettsia [5], Coxiella [6], Brucella [7], Borrelia burgdorferi [8] and Orthonairovirus [9]. Due to its medical importance, $D$. marginatus as many other tick species, has become an emerging threat to the livestock and public health, which needs control measures to mitigate its impact.

Currently, the accessibility and ease of practice have made chemical approach dominant in tick control measures [10]. While the use of conventional acaricides is effective in tick control, the environment is affected by contamination and acaricide residue problems [11]. Furthermore, tick resistance to acaricide has made chemical agents less effective than before [12]. Thus, finding the important target genes of tick species is the key to develop advanced tick control approaches [13, 14].

The research on tick prevention and control methods is greatly facilitated by revealing genome data of tick species. The genome data of Ixodes scapularis Say is the first genomic functional study that provided abundant references to gene annotations of tick species [15]. A very recent study on six tick genomes expounded the comparative genome analyses of ixodid tick species [16]. Together with the I. scapularis genome data, these tick genome data could be of crucial values for reference templates for omics studies such as transcriptome and proteome analyses on ixodid ticks and other tick species [16]. Currently RNA-seq is an effective approach to obtain transcripts on a specific time point. Many transcriptome studies revealed a large number of tick transcripts that were latter used for tick gene function study [17-20], system evolution study [21], and anti-tick vaccine candidate screening $[22,23]$.

Vaccination of livestock is a promising method considering labor-saving, cost-effective for farmers and an ecofriendly approach for mitigation of ticks [24]. But only one available tick vaccine against Bm 86 of Rhipicephalus microplus Canestrini is available, which is less effective against ticks other than $R$. microplus $[25,26]$. New tick-specific antigens are still in need. Now screening of potential protective tick antigens could be achieved by RNA-seq and tick vaccine trials [23]. On the other hand, researches focusing on biological process of tick bloodfeeding and reproduction has enhanced our knowledge on the functions of important tick molecules [27, 28]. According to the study of tick blood meal digestion, Ixodidae ticks has a different way of blood digestion from other hematophagous arthropods by intracellular digestion of hemoglobin [29]. In the process of blood-feeding and digestion of blood meal, there were many genes upregulated encoding proteins, such as ferritin 2 transporting ferric iron in the hemolymph [30], aquaporin regulating body fluid and facilitate salivation [31], and vitellogenin and vitellogenin receptor critical for embryonic genesis [32]. These tick antigens showed potential to compose an anti-tick vaccine.

Besides feeding on hosts, hard ticks live off-host for most of their lives. Ticks conduct sit-and-wait strategy in questing with an extreme endurance to starvation [18]. Ticks initiate different set of genes to cope with longterm starvation and their questing behavior will increase in extended starvation times [18]. During non-feeding period many tick species may express crucial protein for survival that in turn can be harnessed as targets for chemicals. In addition, development of eco-friendly acaricides or tick repellents is also an promising field of tick control [33]. Reports indicated that new acaricide target genes could be used to develop effective but environmentally friendly chemicals to control tick prevalence, for instance, tick kinin receptors regulate numerous tick physiological process including ecdysis and feeding [34].

Regarding $D$. marginatus, the study was in the hope of enriching the molecular information for this tick species and provide a comprehensive viewpoint of this biological system, as current information on $D$. marginatus is limited. Our focus was on three different status of $D$. marginatus female adults as newly molted, starved, and engorged state to conduct an Illumina RNA sequencing (RNA-seq) in order to obtain the transcriptomes of $D$. marginatus female adults. This study of whole tick transcriptome could contribute to understanding of genes involved in blood-feeding and long-term starvation.

\section{Methods}

\section{Tick rearing}

Ticks used in the experiment were F2 generation progeny of a $D$. marginatus adult female originally collected from a 6-year old local-bred female horse in a breeding farm located in Yili prefecture, Xinjiang, China, in April 2018. Ticks were reared under controlled experimental conditions of temperature $\left(24 \pm 1{ }^{\circ} \mathrm{C}\right)$, relative humidity (RH; $90 \pm 5 \%)$ and photoperiod (14 h light: $10 \mathrm{~h}$ dark). The purpose of the RNA-seq analysis was to obtain gene sequences and identify differentially expressed genes of D. marginatus female adults after blood-feeding and long-term starvation regarding the significant role of female adult that plays in feeding large amount of blood and producing thousands of eggs per individual [35]. Another reason choosing females only for the RNA-seq analysis was to minimize the gene expression difference profiled by sexual distinction $[36,37]$.

In order to obtain the $D$. marginatus female adults, we designed three biological groups for RNA-seq analysis (Fig. 1). Larvae $(n \approx 1000)$ and nymphs $(n=400)$ fed on 
the ears of two New Zealand rabbits respectively in succession to become engorged. Subsequently, 363 engorged nymphs successfully molted to adult stage. Adult female ticks after ecdysis for 5 days were designated as $M$ tick group that consisted of 3 biological replicates each made of 5 ticks. Female adult ticks were kept under controlled experimental conditions (as above) for 6 months to become starved ticks as $\mathrm{H}$ tick group that consisted of 3 biological replicates each made of 5 ticks. Finally, $\mathrm{F}$ tick group that consisted of 3 biological replicates each made of 3 engorged female adult ticks was prepared by having 30 starved female adult ticks fed on a local bred yearling for 7 days with 60 male adult ticks around (Fig. 1). The engorged female adult ticks were carefully removed on the 8th day after blood-feeding and kept at controlled experimental conditions (as above) for $24 \mathrm{~h}$ before sample preparation started.

\section{Preparation of the biological samples and total RNA extraction}

Body weight of similar $D$. marginatus female adults were selected to make pooled samples. Sample were prepared as fast as possible to reduce human factors on gene expression. To clean the surface of the ticks, we washed the ticks in phosphate buffer saline (PBS) though mild shaking for $15 \mathrm{~s}$ in the first round. Washing the ticks in $70 \%$ alcohol by the same operation followed next. Last round, wash the ticks in deionized water though mildly shaking for $30 \mathrm{~s}$. Then, after drying the ticks on sterile gauze, ticks that were put in microtubes were rapidly immersed into liquid nitrogen to freeze completely and stored at $-80^{\circ} \mathrm{C}$ for later use.

Total RNA was extracted using Trizol reagent (Invitrogen, Carlsbad, CA, USA) following the manufacturer's procedure. The total RNA quantity and purity were analyzed with Bioanalyzer 2100 (Agilent, Foster, CA, USA) and RNA 1000 Nano LabChip Kit (Nanodrop Technologies, Wilmington, DE, USA) with RIN number $>7.0$. Poly (A) RNA is purified from total RNA $(5 \mu \mathrm{g})$ using poly$T$ oligo-attached magnetic beads using two rounds of purification.

\section{The cDNA library preparation}

After purification, the mRNA was fragmented into small pieces with divalent cations under elevated temperature. Then the cleaved RNA fragments were reverse-transcribed to create the final cDNA library in accordance with the protocol for the mRNA Seq sample preparation kit (Illumina, San Diego, CA, USA), the average size of the insert for the paired-end libraries was $300 \mathrm{bp}$ ( $\pm 50 \mathrm{bp}$ ). Then we performed the paired-end sequencing on an
Illumina Hi-seq 4000 (LC Sciences, USA) following the vendor's recommended protocol.

\section{De novo assembly, unigene annotation and functional classification}

Firstly, Cutadapt [38] and in-house perl scripts were used to remove the reads that contained adaptor contamination, low quality bases and undetermined bases. Then sequence quality was verified using FastQC (http://www. bioinformatics.babraham.ac.uk/projects/fastqc/) including the Q20, Q30 and GC-content of the clean data. All downstream analyses were based on clean data of high quality. De novo assembly of the transcriptome was performed with Trinity v. 2.4.0 [39]. Trinity groups transcripts into clusters based on shared sequence content. The longest transcript in the cluster was chosen as the unigene sequence.

The prediction of coding sequences (>100 amino acids) was performed using TransDecoder, which is part of the Trinity software [40]. All assembled unigenes were aligned against the non-redundant $(\mathrm{Nr})$ protein database (http://www.ncbi.nlm.nih.gov/), Gene ontology (GO) (http://www.geneontology.org), SwissProt (http://www. expasy.ch/sprot/), Kyoto Encyclopedia of Genes and Genomes (KEGG) (http://www.genome.jp/kegg/), Protein family (Pfam) (http://pfam.xfam.org/) and Eggnog (http://eggnogdb.embl.de/) databases using DIAMOND [41] with a threshold of E value $<0.00001$. Local BLAST v. 2.9.0 (ftp://ncbi.nlm.nih.gov/blast/executables/blast + / LATEST/) was used to compare the RNA-seq data of $D$. marginatus with genome sequencing data of $D$. silvarum [16]. Signal peptide prediction of the unigenes encoding proteins was performed with SignalP - 5.0 server (http:// www.cbs.dtu.dk/services/SignalP/).

\section{Differentially expressed unigene analysis}

Salmon [42] was used to perform expression level for unigenes by calculating transcripts per million (TPM) [43]. The differentially expressed unigenes were marked with $\log 2$ (fold change) $>1$ or $\log 2$ (fold change) $<-1$, and gene expression significance was based on BenjaminiHochberg false discovery rate (FDR: $P<0.05)$ and oneway analysis of variance (one-way ANOVA: $P<0.05$ ) by R package edgeR [44]. GO and KEGG enrichment analysis were again performed on the differentially expressed unigenes by in-house perl scripts.

\section{Real-time quantitative PCR validation of RNA-seq results}

The consistency of the gene expression levels was confirmed by real-time quantitative PCR. The RT-qPCR was performed on another set of samples in a separate tick 


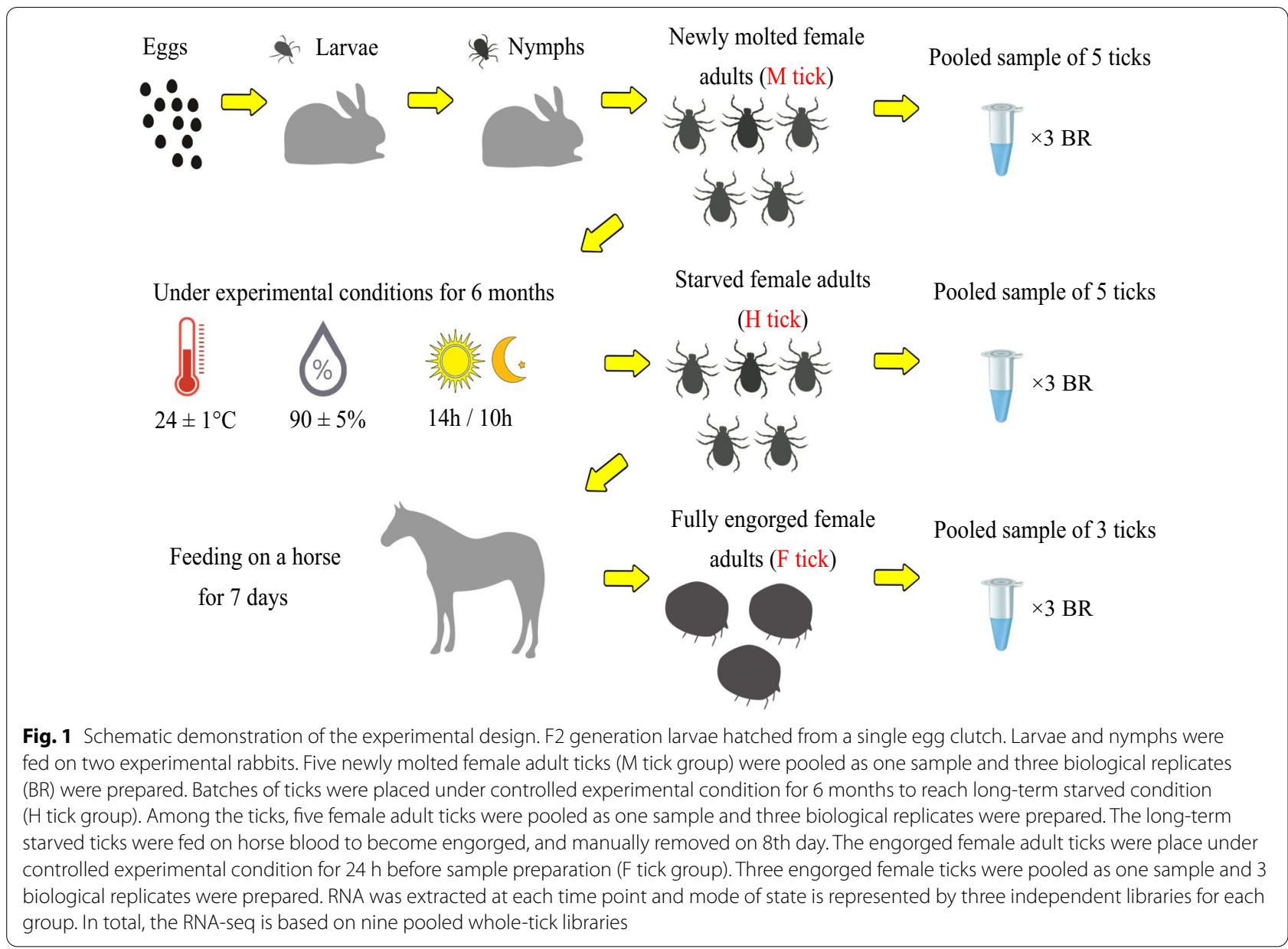

feeding experiment with the same sample preparation procedure above-mentioned. and the expression levels of 6 particular genes were assessed. Ferritin 1 (DN38021), Ferritin 2 (DN51538), HSP 70 (DN48195), galectin (DN57588), glutathione S-transferase (DN38169), and Dm86 (DN57937) were selected among the genes differentially expressed in newly molted ( $M$ tick), starved ( $\mathrm{H}$ tick), and fed female ticks (F tick). RNA extraction procedure was performed as described above. The first strand cDNA templates were synthesized using a Fastking RT cDNA synthesis kit (Tiangen Biotech, Beijing, China). Single-strand cDNA was used, and transcription profile was conducted through real-time PCR using QuantiNova SYBR Green PCR Kit (Qiagen, Hilden, Germany) with an Applied Biosystems 7500 Fast Real-Time PCR System (Applied Biosystems, Foster, USA). Specific primers for target gene and the internal control are shown in Additional file 1: Table S1. The RT-qPCR procedure started with initial denaturation at $95{ }^{\circ} \mathrm{C}$ for $2 \mathrm{~min}$, followed by 40 cycles of a denaturation step at $95^{\circ} \mathrm{C}$ for $10 \mathrm{~s}$ and an annealing/extension step at $60{ }^{\circ} \mathrm{C}$ for $35 \mathrm{~s}$. The mRNA levels of the analyzed genes both in transcriptome and
RT-qPCR were normalized using the elongation factoralpha 1 (ef- $\alpha 1)$ gene of D. marginatus (DN43634) using the comparative $\mathrm{Ct}$ method [45].

\section{Results and discussion}

Sequencing and assembly

Illumina sequencing of the nine $D$. marginatus adult female tick samples resulted in 460,171,414 raw reads (Additional file 2: Table S2). After removing low quality reads, 449,349,046 valid reads accounted for $97.68 \%$ of the raw reads. De novo transcriptome assembly was performed using Trinity software, resulting in a total of 100,644 putative transcripts (N50 1383), clustered into 30,251 unigenes. A summary of the assembly is shown in Table 1 . Regarding sequence length, all unigenes are longer than $200 \mathrm{bp}$. The longest is $24,550 \mathrm{bp}$. Fifteen percent of assembled unigenes were above 2000 bp, and many of the unigenes were between 300-1000 bp (39.04\%) (Fig. 2a). Principal components analysis (PCA) of the assembly revealed biological replicates grouped together separately showing acceptable variation within groups (Fig. 2b). It is notable that this is the 
first transcriptome analysis of $D$. marginatus produced using RNA-Seq. The assembly results of this study have augmented sequence information of $D$. marginaus. The transcriptome data used in this analysis were submitted to GEO (Gene Expression Omnibus, http://www.ncbi. nlm.nih.gov/geo/) database of NCBI under the accession number GSE151667.

\section{Annotation}

Functional annotation of the assembled unigenes were performed by matching the 30,251 unigene sequences against 6 databases. $\mathrm{Nr}$ database hit the highest match up to $32 \%$, while Swissprot database hit $22.56 \%$, relatively less than the other database (Additional file 3: Table S3). Among the 9672 annotated unigenes, $58.9 \%$ of them showed homology with sequences of tick species, and over half of the unigenes matched $I$. scapularis sequences (Fig. 3). The remaining 20,579 unigenes (68.03\%) were classified as unknown because they did not produce matches within the searched databases, indicating that many new genes and non-coding RNA sequences had been identified $[37,46]$. Genome data is very important to tick transcriptome studies. Recently published tick genome study described 6 tick species that are common in the Old Continent [16]. These genome data are valuable in studying tick gene function, system evolution, and symbiotic relationship of the pathogen in different tick species. Among the genome data, Dermacentor silvarum Olenev is a closely related tick species with $D$. marginatus. The two tick species are morphologically similar, but surprisingly genomic alignments of the $D$. marginatus RNA-seq data with genome sequencing data of $D$. silvarum showed that the two tick species did not show very high sequence similarity (Additional file 4: Table S4). The reason that most of hits matched with $I$. scapularis rather than $D$. silvarum was probably because sequence information on I. scapularis is abundant in the searched databases currently [15]; nevertheless, the $D$. silvarum genome sequencing data would be soon available as reference for annotation in the databases. We performed a Local BLAST to compare the RNA-seq data of D. marginatus with genome sequencing data of $D$. silvarum, and the results revealed that 5841 out of 9573 unigenes with coding regions (CDs) matched that of genome sequencing data of $D$. silvarum with varying degrees. For identifying potential tick proteins, the SignalP prediction was performed and the results indicated that 320 out of 9573 unigenes encoding proteins have signal peptide sequence, while 150 were among the unidentified unigenes. The SignalP prediction results indicated that many potential

Table 1 Summary of the D. marginatus transcriptome assembly

\begin{tabular}{llllll}
\hline Index & All & Min length & Mid length & Max length & Total assembled bases \\
\hline Transcript & 100,644 & 201 & 509 & 24,550 & $86,647,606$ \\
Unigene & 30,251 & 201 & 628 & 24,550 & $31,889,293$ \\
\hline
\end{tabular}

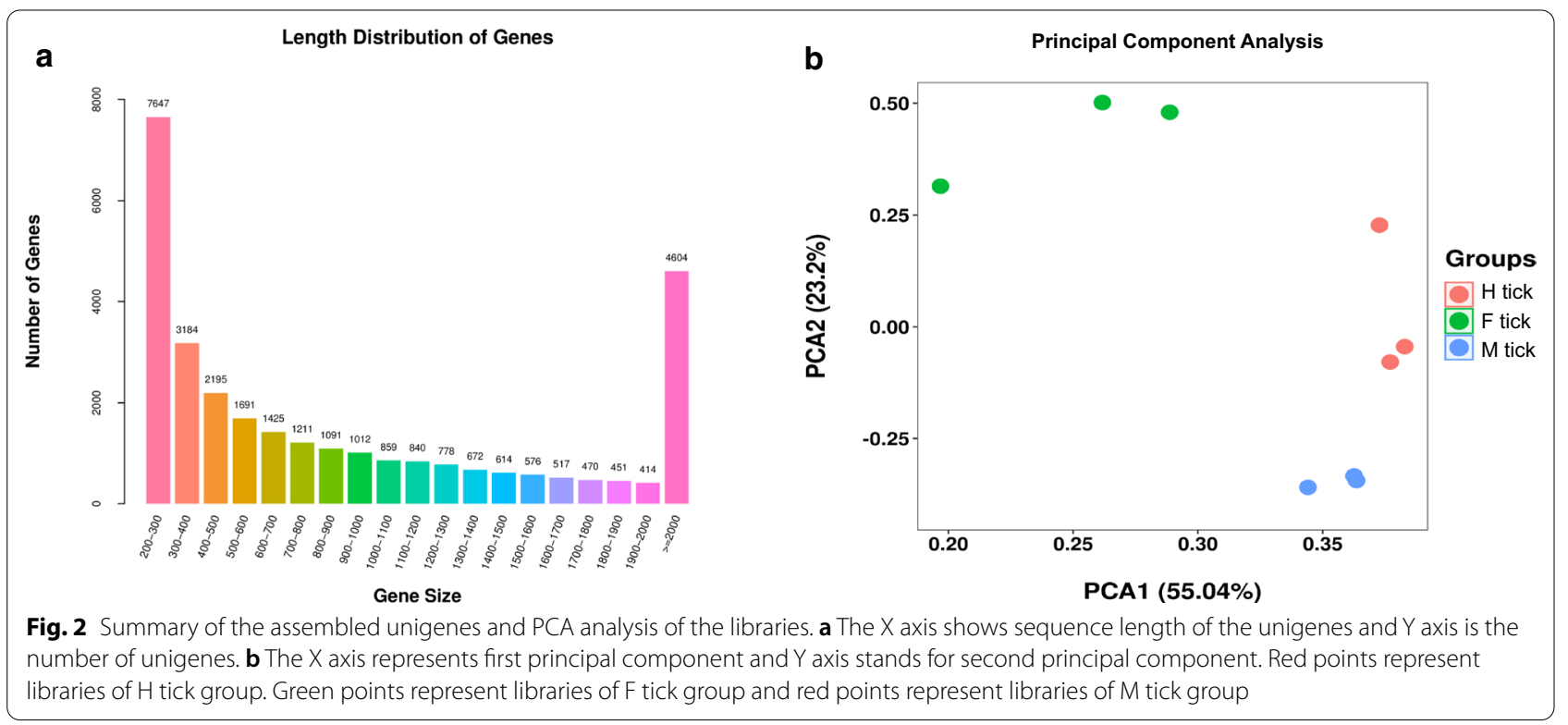


tick genes encoding proteins are still waiting to be uncovered. The unigene annotation table, sequence alignment results and the signal peptide prediction results were incorporated as Additional file 5: Table S5, and the information contained in the table could be helpful in finding potential tick gene orthologs and proteins.

\section{Gene Ontology (GO) classification}

The annotated sequences were next functionally characterized using the information available in GO classification that considers 3 categories. Initially, genes were classified according to their molecular function, cellular component, and biological process using the GO terms from the GO database (Fig. 4). Only the categories represented by more than 30 genes are depicted, each including the number of the genes assigned.

The most abundantly annotated functions were binding activity, such as protein binding activity $(n=211)$, ATP binding activity $(n=185)$ and metal ion binding $(n=183)$ which coincided with transcriptomes of other ixodid tick species [47, 48]. The remaining annotated functions were as follows: structural constituent of ribosome $(n=210)$, RNA binding $(n=182)$, oxidoreductase activity $(n=170)$, zinc ion binding $(n=125)$, DNA binding $(n=121)$, hydrolase activity $(n=106)$, nucleic acid binding $(n=98)$.

Gene classification according to cellular component resulted in up to 15 categories represented by more than 100 genes. In the cellular component category, the most abundant GO term was cytoplasm, assigned to $17.1 \%$ $(n=664)$ of the total GO annotated unigenes. Many GO terms related to nucleus were abundant either $(n=555)$. Membrane $(n=327)$ and integral component of membrane structure $(n=390)$ followed next.

Gene classification based on biological process resulted in 25 categories. Notably, the most abundant $(n=171)$ was translation process followed by categories corresponding to oxidation-reduction process $(n=141)$, proteolysis $(n=120)$, regulation of transcription $(n=91)$, and metabolic process $(n=90)$. The remaining annotations of biological process that contained less unigenes were distributed into twenty categories. The GO terms for molecular function, cellular compartment and biological process assigned to all the genes annotated are showed in Additional file 6: Table S6.

\section{Differential gene expression profiles}

Blood-feeding and long-term starvation caused significant differential gene expression of $D$. marginatus. The volcano plots were applied to demonstrate the overall gene expression profiles (Fig. 5). Differentially expressed genes (DEGs) were almost one third of the overall unigenes and the downregulated unigenes slightly outnumbered upregulated unigenes between each compared group (Additional file 7: Figure S1) indicating the three different states of $D$. marginatus female adults were experiencing significant physiological function shift which was in line with many ixodid transcriptome studies $[18,23,49]$. It is considered that $\mathrm{H}$ tick represents starved ticks that experienced 6 months non-feeding period exhausting its energy sources of lipid, carbohydrate, and protein, whereas $M$ tick stands for newly molted ticks with a physiological status at its prime with many of the energy metabolism-related genes downregulated [18]. After mating, a $D$. marginatus female adult could ingest over 100 -fold on its body weight in blood [35]. The blood meal is digested and the nutrients are transported and mostly used for embryogenesis [27]. Both blood-feeding and long-term starvation would cause significant differential gene expression.

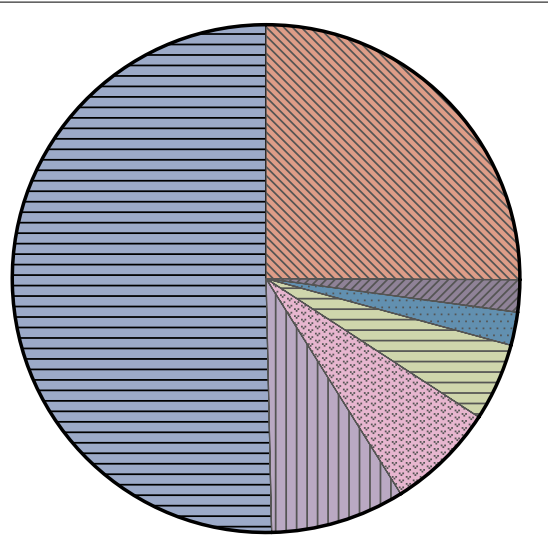

$\begin{array}{ll}\square 50.35 \%, 4870 & \text { Ixodes scapularis } \\ \square 8.55 \%, 827 & \text { Other tick species } \\ \square 6.92 \%, 669 & \text { Limulus polyphemus } \\ \square 4.95 \%, 479 & \text { Centruroides sculpturatus } \\ \square 2.11 \%, 204 & \text { Parasteatoda tepidariorum } \\ \square 2.05 \%, 198 & \text { Stegodyphus mimosarum } \\ \square 25.07 \%, 2425 & \text { Other }\end{array}$

Total $=100 \%, 9672$

Fig. 3 The pie chart of taxonomic assignment of unigenes. The percentage of unigenes assigned to each taxonomic group is shown on the right. Best hits to "Other" include arthropods other than tick species 


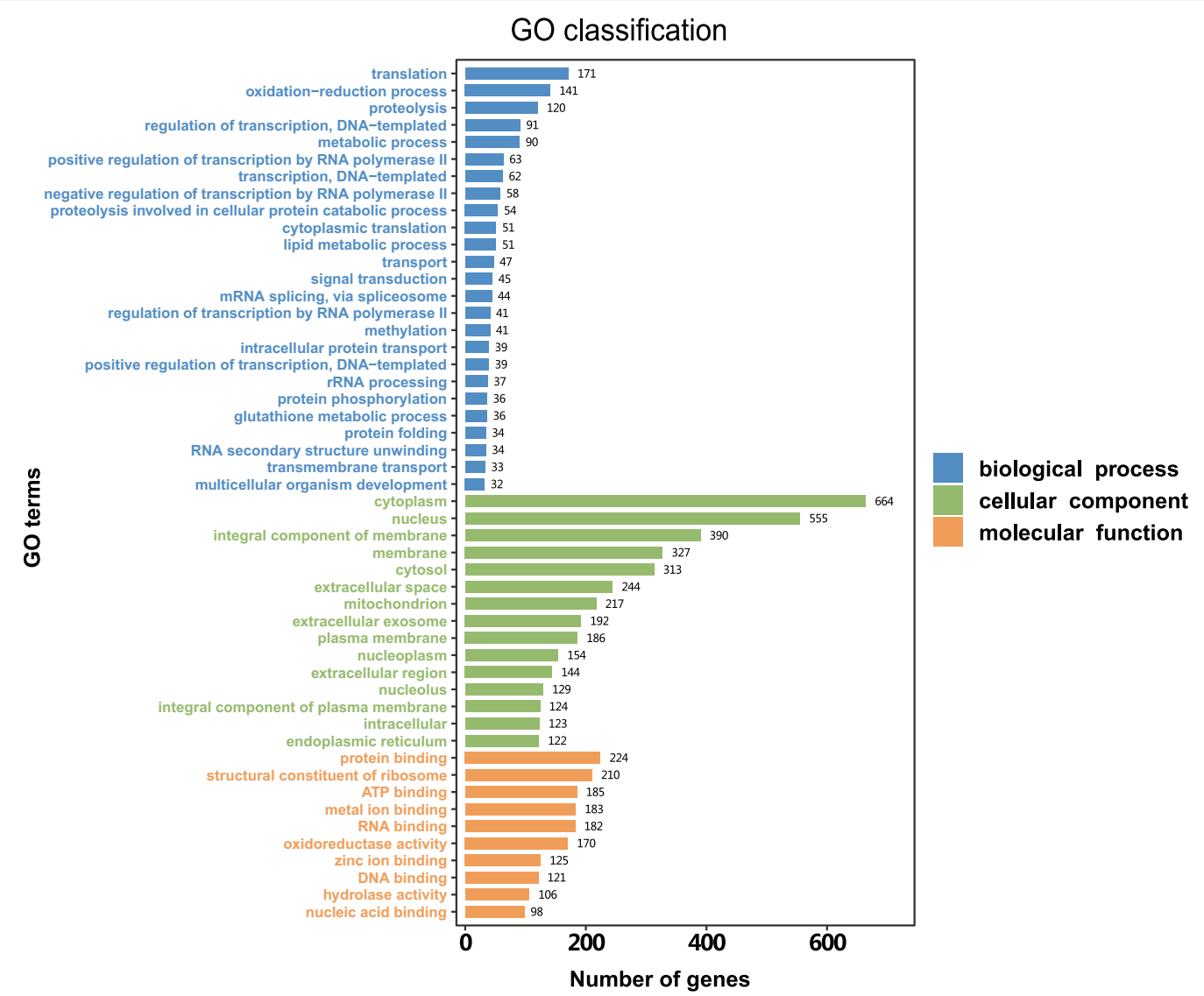

Fig. 4 Gene Ontology classification of D. marginatus transcriptome data. Bars and numbers represent number of genes in each protein class. Blue bars refer to biological process, green bars refer to cellular component and orange bars refer to molecular function. The X axis shows the number of unigenes in each category

For further interpretation of systemic functions, the $D$. marginatus dataset was mapped to KEGG pathways. The 15 pathways most enriched in specific stages of female adult ticks are presented in Fig. 6. The significantly enriched pathways consisted of protein processing, glycan biosynthesis, lipid metabolism, detoxification, and defense responses.

Currently it is accepted that proteins in the blood meal are the main source of nutrient for oviposition and the energy source for resisting long-term starvation $[18,19$, 50]. Upregulated genes in the three stages potentially involved in protein metabolism are listed in Table 2 and Additional file 5: Table S5. Protein families of proteases, such as cysteine peptidases, aspartic endopeptidases, metallopeptidases and serine peptidases were significantly upregulated in the F tick group (Fig. 6a) suggesting that the proteolytic system was active [46]. The results are quite similar to other transcriptome studies [22,37]. The intracellular digestion of the proteins ingested with blood is performed by a group of lysosomal proteolytic enzymes that act sequentially and have been studied in detail in the family Ixodidae [27, 29, 51, 52]. It has been demonstrated that haemoglobin is the source of haem group for the development of the embryo in ixodids, and it is essential for a successful hatching of eggs spawned by engorged female ticks [22]. In the $\mathrm{H}$ tick group proteolysis enhanced, and pathways associated with diseases were underlined (Fig. 6c). In starvation, there was an upregulation of pathways related to transcription and translation processes that are energetically expensive. The result is in consistence with a previous study on Dermacentor variabilis Say [18]. It was probably caused by excessive consumption of nutrient substances so as to expend proteins in its cellular structure to maintain basic life support [18]. In the $M$ tick group, pathways of steroid biosynthesis, protein export and N-Glycan biosynthesis were significantly enriched (Fig. 6b). New adult ticks emerging from engorged nymphs have consumed their last meal taken in their nymphal state. Host blood components, especially hemoglobin, were digested, and subsequently biosynthesized for new proteins acting on hormones and chitin biosynthesis [53]. As tick experience long-term 


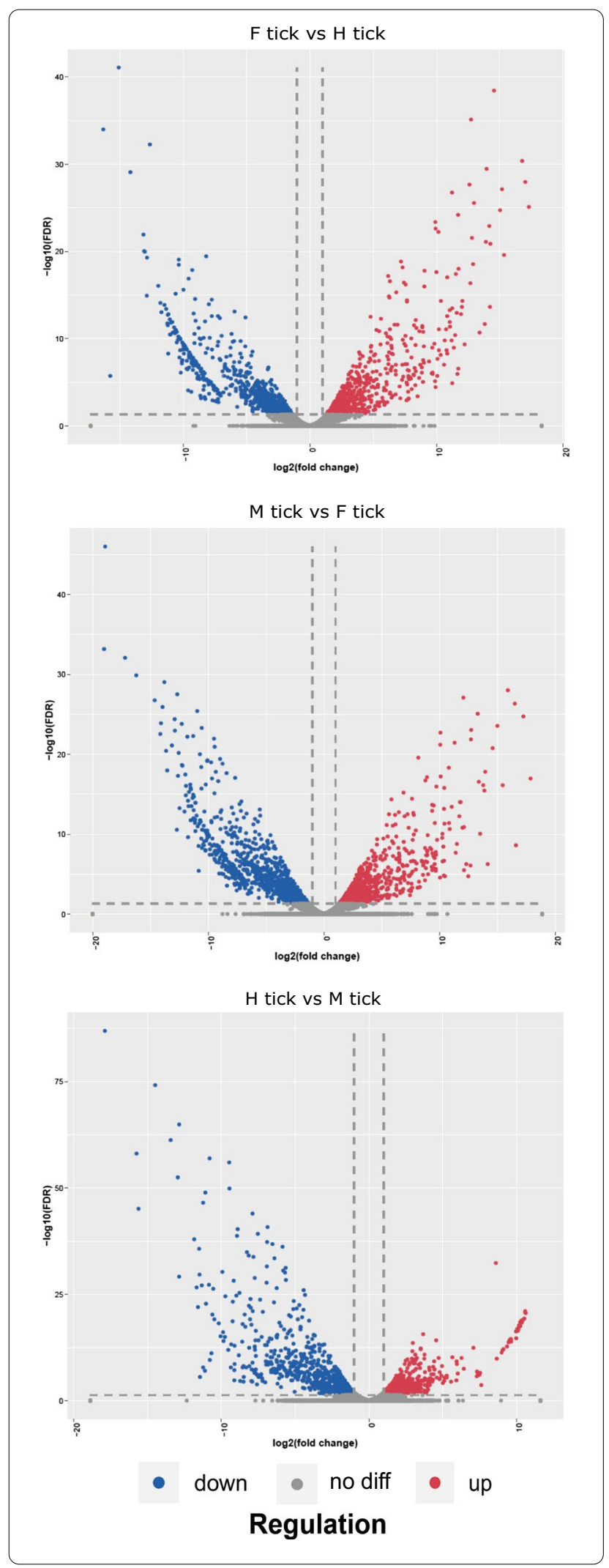

Fig. 5 Scatter plots of the gene expression counts between groups. The $X$ axis shows $\log 2$ fold-change that is plotted against the mean expression of each unigese of the two groups. Y axis is - $\log 10$ FDR value (Student's t-test, $P<0.05$ ) shown from 0 to 80 . Every point represents one transcript. Differentially expressed transcripts having a fold change of $>1.5$ and $P<0.05$ (Student's t-test) are represented by red and blue dots. Gray dots represent Genes not differentially expressed

starvation, the energy sustaining life comes mainly from consumption of proteins in tick midgut $[18,54,55]$.

In invertebrates, lipids perform several functions including serving as constituents of cellular structures, hormones, energy, and play roles in egg production and metamorphosis [56, 57]. Lipid metabolism is of crucial importance in D. marginatus, approximately 150 unigenes annotated were identified with a putative function in lipid metabolism. We selected 49 unigenes that are differentially expressed in blood-feeding and starvation (Table 3, Additional file 5: Table S5). The KEGG pathway enrichment revealed that sphingolipid metabolism, synthesis and degradation of ketone bodies, steroid hormone biosynthesis, arachidonic acid metabolism were related to lipid metabolism after blood-feeding. The unigene annotated as vitellogenin (DN57762) in the F tick group was significantly upregulated compared to both the $\mathrm{H}$ tick and $\mathrm{M}$ tick group. Vitellogenin is essential for egg development and oviposition, and has been shown to play a role in heme sequestration $[58,59]$. In D. variabilis, it has been demonstrated that Vg expression increases after engorgement and $\mathrm{Vg}$ is exclusively expressed in fat body and gut cells of vitellogenic females but not in the ovary [60]. Beside vitellogenin, several apolipoproteins belong to the low-density lipoprotein family were upregulated. Corresponding to the lipoprotein, the low-density lipoprotein receptor (LDLR) was upregulated as well during blood-feeding, which is the major cholesterol-carrying lipoprotein of plasma, acting to regulate cholesterol homeostasis in cells [61]. In ticks, the LDLR binds LDL and transports it into digestive cells [62]. It has been considered that arthropods lack the set of enzymes to synthesize cholesterol, so they are obliged to obtain cholesterol from their hosts [63]. The unigenes involved in cholesterol metabolism were insulin induced protein (DN55975), high-density lipoprotein (DN55629), Niemann-Pick type C1 (DN34326) and Niemann-Pick type C2 (DN53284) during blood-feeding, and their expression profile indicated lipid metabolism was very active in newly molted ticks. In non-ruminants, the INSIG1 gene modulates cholesterol metabolism, lipogenesis, and glucose homeostasis with a cellular localization on endoplasmic reticulum membrane [64]. High density 

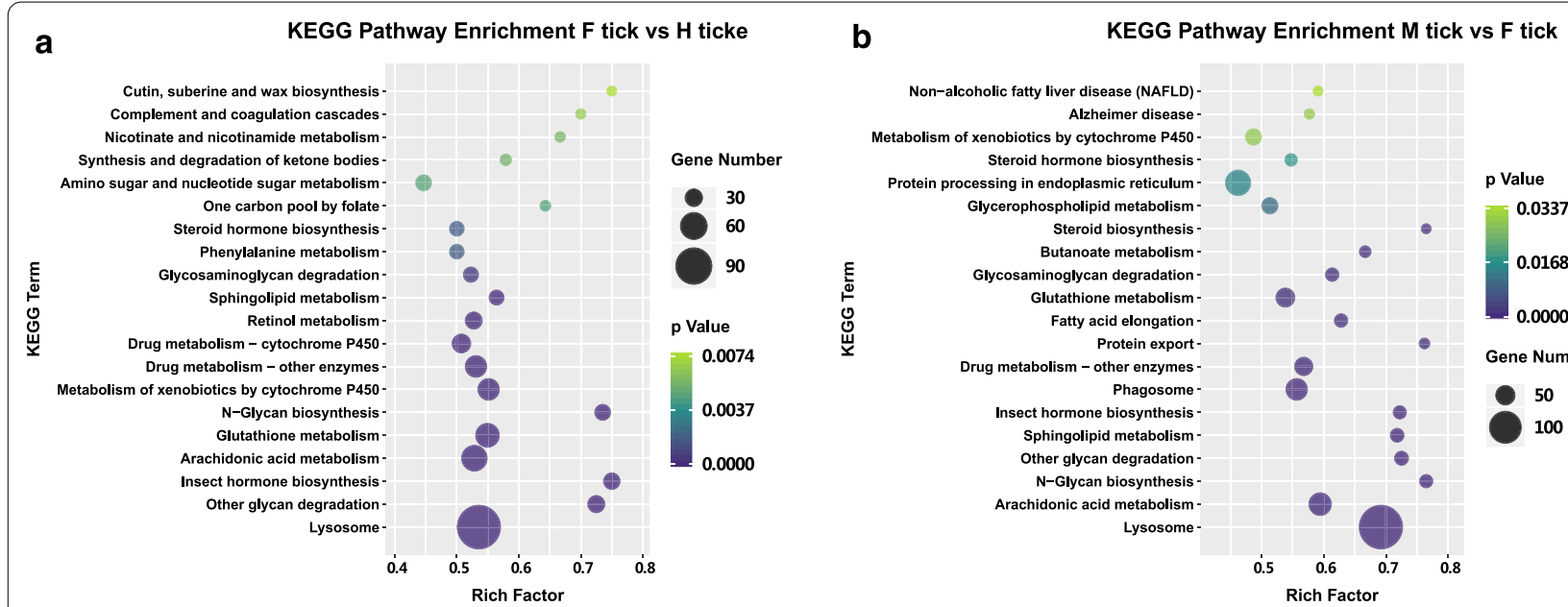

C

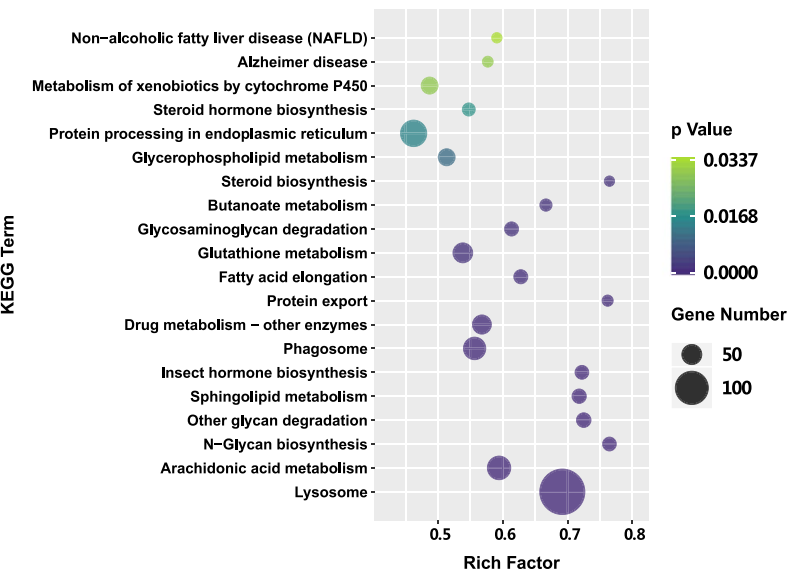

KEGG Pathway Enrichment $H$ tick vs M tick

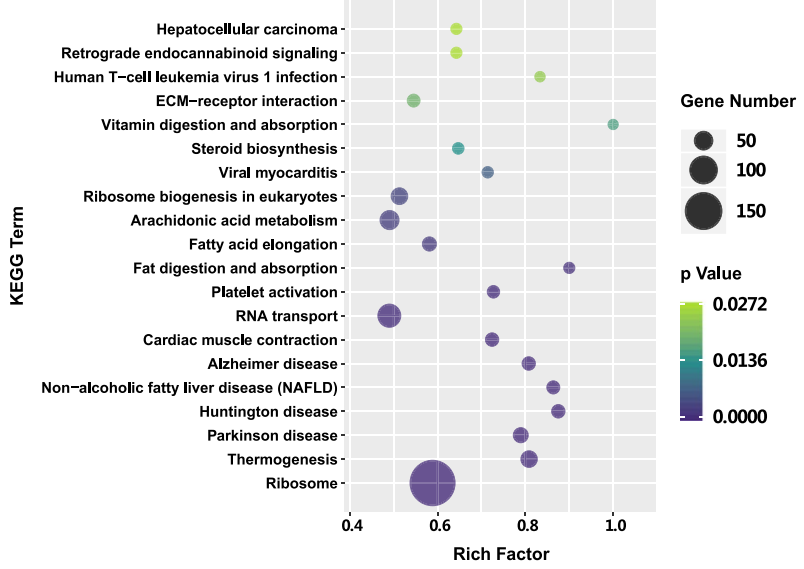

Fig. 6 Significantly enriched KEGG pathways associated with blood-feeding and starvation. a Pathways enrichment between $\mathrm{F}$ tick and $\mathrm{H}$ tick. $\mathbf{b}$ Pathways enrichment between $\mathrm{M}$ tick and F tick. c Pathways enrichment between $\mathrm{H}$ tick and $\mathrm{M}$ tick. The top 20 most significant KEGG terms were illustrated for each compared pair. The $X$ axis shows the rich factor that is the ratio of differentially expressed unigenes in a pathway term to all gene numbers annotated in the same pathway term. The bubbles represent the number of unigenes and the gradient coloration indicates the corrected $P$-value by the FDR method. Only pathways with $P<0.05$ were shown

lipoprotein transports diglyceride from the fat body cell into the hemolymph, and is involved in the transport of cholesterol from the gut into the hemolymph in insects $[65,66]$. Niemann-Pick type C1 encodes a large membrane glycoprotein with mostly a late endosomal localization. Niemann-Pick type C2 encodes a small soluble lysosomal protein with high affinity binding to cholesterol. Both proteins are in intracellular regulation of cholesterol metabolism, and their efficiency determine the processing and utilization of endocytosed cholesterol [67]. Many unigenes related to sphingolipid metabolism were upregulated after blood-feeding (Table 3). Sphingolipids are one of the major classes of eukaryotic lipids and were appreciated as components of the plasma membrane and as modulators of cell-cell interactions and cell recognition [68]. Other upregulated unigenes involved in lipid transport were the oxysterol-binding protein, hemelipoglycoprotein, fatty-acid binding protein, microsomal triglyceride transfer protein (Table 3), in which several were upregulated after long-term starvation. Unlike absorption of lipids during blood-feeding, the female adult ticks undergoing long-term starvation were in steady consumption of lipid reserves [18]. Compared to newly molted and engorged ticks, starved ticks presented most of the genes downregulated regarding lipid metabolism, whereas, a number of genes related to hormone synthesis and wax formation were upregulated in the $\mathrm{H}$ tick and $\mathrm{M}$ tick groups indicating these genes might play a role in survival of $D$. marginatus during the off-host period. 
Table 2 Selected differentially expressed unigenes putatively involved in protein metabolism after blood-feeding and long-term starvation

\begin{tabular}{|c|c|c|c|c|c|c|}
\hline Predicted Function & Annotation & TPM F-tick & TPM H-tick & TPM M-tick & ANOVA ( $P$-value) & Unigene \\
\hline \multirow[t]{7}{*}{ Proteinase } & Midgut cysteine proteinase & 250.11 & 105.93 & 9.57 & 0.02 & DN42765 \\
\hline & Hypothetical protein & 244.25 & 0.71 & 0.01 & 0 & DN53495 \\
\hline & Conserved hypothetical protein & 0 & 69.91 & 14.74 & 0 & DN35517 \\
\hline & Putative serine protease & 58.94 & 84.12 & 16.04 & 0 & DN46725 \\
\hline & Midgut cysteine proteinase 2 & 139.38 & 142.84 & 27.02 & 0 & DN40266 \\
\hline & Astricsin & 0 & 42.25 & 0 & 0 & DN38987 \\
\hline & Conserved hypothetical protein & 0.60 & 17.25 & 2.04 & 0 & DN56731 \\
\hline \multirow[t]{7}{*}{ Metalloprotease } & Leucine aminopeptidase & 125.81 & 21.76 & 9.79 & 0 & DN46003 \\
\hline & Conserved hypothetical protein & 233.58 & 60.91 & 36.54 & 0 & DN58308 \\
\hline & Astacin-like metallopeptidase & 10.37 & 2.76 & 0.60 & 0 & DN37813 \\
\hline & Neprilysin & 6.01 & 45.58 & 6.32 & 0 & DN54652 \\
\hline & Astacin-like metalloprotease & 8.29 & 0.05 & 0.04 & 0 & DN23285 \\
\hline & Membrane metallo-endopeptidase & 11.90 & 0.19 & 0.27 & 0 & DN54193 \\
\hline & Zinc metalloprotease & 6.17 & 26.69 & 2.60 & 0 & DN56686 \\
\hline \multirow[t]{2}{*}{ Cathepsin L } & Cathepsin L-like cysteine proteinase & 441.24 & 427.17 & 84.50 & 0 & DN56942 \\
\hline & Cathepsin L & $1,254.14$ & 141.31 & 57.16 & 0 & DN40829 \\
\hline \multirow[t]{2}{*}{ Cathepsin B } & Cathepsin B endopeptidase & 522.98 & 106.56 & 12.00 & 0 & DN54757 \\
\hline & Cathepsin B & 65.82 & 46.24 & 11.38 & 0 & DN52552 \\
\hline Cathepsin C & Cathepsin C precursor & 148.74 & 222.46 & 47.97 & 0 & DN42108 \\
\hline \multirow[t]{4}{*}{ Cathepsin D } & Yolk cathepsin & 138.57 & 0.05 & 0.04 & 0 & DN56458 \\
\hline & Aspartic protease & 58.94 & 0.30 & 0.04 & 0 & DN33194 \\
\hline & Heme-binding aspartic proteinase & 146.34 & 0.11 & 0.05 & 0 & DN36019 \\
\hline & Aspartic protease & 35.25 & 127.92 & 10.05 & 0 & DN44103 \\
\hline \multirow[t]{2}{*}{ Carboxypeptidase } & Carboxypeptidase & 3.50 & 0.08 & 23.44 & 0 & DN57960 \\
\hline & Conserved hypothetical protein & 65.18 & 0.03 & 0.00 & 0 & DN54491 \\
\hline \multirow[t]{4}{*}{ Serine protease inhibitor } & Serine protease inhibitor 3 & 199.21 & 9.24 & 16.00 & 0 & DN39339 \\
\hline & Serine protease inhibitor 17 & 178.14 & 9.03 & 24.05 & 0 & DN54400 \\
\hline & Serine protease inhibitor 12 & 27.37 & 3.68 & 44.21 & 0 & DN53734 \\
\hline & $\begin{array}{l}\text { Kunitz/Bovine pancreatic trypsin } \\
\text { inhibitor Domain protein }\end{array}$ & $1,274.39$ & 0.07 & 0.02 & 0 & DN56779 \\
\hline \multirow[t]{3}{*}{ Autophagy-related protein } & Autophagy-related protein 16 & 3.39 & 9.07 & 3.64 & 0.04 & DN40035 \\
\hline & Autophagy-related protein $9 \mathrm{~A}$ & 7.65 & 9.52 & 6.28 & 0.32 & DN55372 \\
\hline & Autophagy-related protein 12 & 24.88 & 35.90 & 22.76 & 0.13 & DN37604 \\
\hline
\end{tabular}

TPM values are the mean of the three biological replicates analyzed from each physiological condition. Significance analysis was based on one-way ANOVA $(P<0.05)$. Full information on unigene names, TPM data, and other information see Additional file 5: Table S5

Carbohydrates, as proteins and lipids ingested with host blood, are the essential nutrients for ticks. A recent study demonstrated the major pathways involved in carbohydrate metabolism with details indicating blood glucose is an important nutrient for I. scapularis [20]. In the $D$. marginatus transcriptome analyzed in this study, several carbohydrases, carbohydrate transporters and cuticular proteins were identified (Table 4, Additional file 5: Table S5). Up to 55 unigenes on carbohydrate metabolism were differentially expressed (35 in the $\mathrm{F}$ tick group, 9 in the $\mathrm{H}$ tick group and 11 in the $\mathrm{M}$ tick group), covering biological processes such as carbohydrate phosphorylation, protein glycosylation, glycogen catabolic process, galactose metabolic process. The results indicated that the upregulated unigenes encoding carbohydrate-metabolizing enzymes were similar with the genes identified in the transcriptomes of Ixodes ricinus Linnaeus [17], Haemaphysalis flava Neumann [37], D. variabilis [69], and the argasids Ornithodoros moubata Murray [46] and Ornithodoros erraticus Neumann [63]. According to the annotations, the upregulated unigenes were associated with the metabolism and transport 
Table 3 Selected differentially expressed unigenes putatively involved in lipid metabolism after blood-feeding and long-term starvation

\begin{tabular}{|c|c|c|c|c|c|c|}
\hline Protein/Function & Annotation & TPM F-tick & TPM H-tick & TPM M-tick & ANOVA ( $P$-value) & Unigene \\
\hline \multirow[t]{6}{*}{ Lipoprotein } & Vitellogenin & $3,180.28$ & 0.06 & 0.07 & 0 & DN57762 \\
\hline & Vitellogenin-B & 301.42 & 336.42 & 40.48 & 0 & DN58267 \\
\hline & Low-density lipoprotein receptor-related protein & 0.08 & 4.18 & 0.47 & 0 & DN38842 \\
\hline & Ixodes scapularis hypothetical protein & 46.51 & 19.10 & 4.59 & 0 & DN58114 \\
\hline & Apolipoprotein & 0.00 & 0.08 & 19.97 & 0 & DN37679 \\
\hline & Apolipoprotein & 0.00 & 1.52 & 41.34 & 0 & DN34916 \\
\hline \multirow[t]{4}{*}{ LDL-receptor } & Lipophorin receptor, putative & 43.28 & 20.04 & 9.58 & 0 & DN55485 \\
\hline & LDL-receptor class A domain-containing protein & 15.24 & 4.61 & 10.37 & 0 & DN58047 \\
\hline & LDL-receptor class A domain-containing protein & 18.39 & 0.29 & 3.81 & 0 & DN50871 \\
\hline & LDLR chaperone boca-like & 29.84 & 21.93 & 7.41 & 0.01 & DN56139 \\
\hline \multirow[t]{2}{*}{ Acyl-CoA synthetase } & Acyl-CoA synthetase family member & 33.65 & 2.18 & 0.51 & 0 & DN42480 \\
\hline & Acyl-CoA synthetase family member & 0.12 & 14.37 & 33.01 & 0 & DN56802 \\
\hline \multirow[t]{5}{*}{ Cholesterol metabolism } & Insilin induced protein & 8.99 & 7.13 & 13.76 & 0.02 & DN55975 \\
\hline & Putative high-density lipoprotein-binding protein & 103.71 & 19.57 & 4.93 & 0 & DN55629 \\
\hline & Fatty acyl-CoA elongase & 62.53 & 7.57 & 8.65 & 0 & DN57046 \\
\hline & Fatty acyl-CoA elongase, putative & 47.18 & 7.82 & 14.56 & 0 & DN33724 \\
\hline & Fatty acyl-CoA elongase, putative & 101.92 & 22.09 & 93.38 & 0.01 & DN45177 \\
\hline \multirow[t]{3}{*}{ Niemann-Pick type C1 } & Niemann-Pick type C1 domain-containing protein & 43.49 & 19.39 & 11.53 & 0 & DN50927 \\
\hline & Putative Niemann-Pick type C1 domain-containing protein & 35.83 & 6.11 & 18.27 & 0 & DN34654 \\
\hline & Putative Niemann-Pick type $\mathrm{C} 1$ domain-containing protein & 176.11 & 0.29 & 0.03 & 0 & DN34326 \\
\hline Niemann-Pick type C2 & Major epididymal secretory protein HE1, putative & 33.38 & 0.80 & 8.31 & 0 & DN53284 \\
\hline \multirow[t]{2}{*}{ Hemelipoglycoprotein } & Hemelipoglycoprotein precursor & $1,247.69$ & 0.78 & 0.50 & 0 & DN45062 \\
\hline & Hemelipoglycoprotein precursor & 5.69 & 0.21 & 1.22 & 0 & DN58109 \\
\hline \multirow[t]{3}{*}{ Lipid transporter } & Predicted microsomal triglyceride transfer protein & 171.76 & 19.53 & 5.56 & 0 & DN43048 \\
\hline & Fatty-acid and retinol-binding protein 1 & 0.00 & 6.56 & 0.00 & 0 & DN59148 \\
\hline & Lipid droplet-associated hydrolase & 0.20 & 9.14 & 0.45 & 0 & DN48962 \\
\hline \multirow[t]{2}{*}{ Oxysterol-binding protein } & Oxysterol-binding protein-related protein 2 & 14.81 & 48.91 & 13.96 & 0 & DN54120 \\
\hline & Oxysterol-binding protein-related protein 9 & 7.03 & 28.49 & 14.11 & 0.01 & DN54199 \\
\hline Fatty acid-binding protein & Fatty acid-binding protein & $1,056.66$ & 724.66 & 357.63 & 0 & DN48942 \\
\hline \multirow[t]{2}{*}{ Glycerol kinase } & Lycerol kinase-like isoform X2 & 6.08 & 11.88 & 2.60 & 0.01 & DN47773 \\
\hline & Mitochondrial-like acylglycerol kinase & 8.20 & 14.71 & 3.36 & 0.01 & DN50098 \\
\hline \multirow[t]{5}{*}{ Sphingolipid metabolism } & Putative shingomyelin phosphodiesterase & 17.53 & 0.13 & 0.13 & 0 & DN45324 \\
\hline & Sphingomyelin phosphodiesterase 4-like isoform X1 & 6.38 & 22.13 & 8.08 & 0.02 & DN55541 \\
\hline & Putative beta-glucocerebrosidase & 114.00 & 0.05 & 0.26 & 0 & DN35305 \\
\hline & Putative beta-glucocerebrosidase & 494.63 & 0.21 & 0.05 & 0 & DN53737 \\
\hline & Conserved hypothetical protein & 5.51 & 8.20 & 1.59 & 0.01 & DN56056 \\
\hline \multirow[t]{3}{*}{ Ecdysone synthesis } & Ecdysone-induced protein 75 & 4.98 & 35.95 & 13.26 & 0 & DN40090 \\
\hline & Ecdysone-induced protein 63F1 & 1.51 & 16.91 & 6.71 & 0 & DN38040 \\
\hline & Conserved hypothetical protein & 7.24 & 0.11 & 0.02 & 0 & DN38433 \\
\hline \multirow[t]{2}{*}{ Phospholipase } & Phospholipase $\mathrm{A} 2$ precursor, putative & 36.94 & 0.08 & 0.13 & 0 & DN31517 \\
\hline & Phospholipase A2 precursor, putative & 194.59 & 29.04 & 41.49 & 0 & DN56186 \\
\hline \multirow[t]{2}{*}{ Gastric triacylglycerol lipase } & Gastric triacylglycerol lipase, putative & 9.9 & 0.31 & 0.82 & 0 & DN47082 \\
\hline & Gastric triacylglycerol lipase, putative & 26.2 & 7.34 & 0.93 & 0 & DN52640 \\
\hline \multirow[t]{6}{*}{ Cuticular wax formation } & Acyl-CoA reductase, putative & 2.68 & 2.25 & 400.44 & 0 & DN56552 \\
\hline & Acyl-CoA reductase, putative & 24.53 & 0.22 & 0 & 0 & DN52493 \\
\hline & Acyl-CoA reductase, putative & 23.75 & 0.03 & 0 & 0 & DN43844 \\
\hline & Acyl-CoA reductase, putative & 27.14 & 0.91 & 0.02 & 0 & DN51418 \\
\hline & Acyl-CoA reductase, putative & 47.33 & 1.04 & 0.87 & 0 & DN43844 \\
\hline & Acyl-CoA reductase, putative & 2.82 & 12.26 & 4.74 & 0 & DN53780 \\
\hline
\end{tabular}

TPM values are the mean of the three biological replicates analyzed from each physiological condition. Significance analysis was based on one-way ANOVA $(P<0.05)$. Full information on unigene names, TPM data, and other information see Additional file 5: Table S5 
of several carbohydrates including glucose, fructose, mannose, galactose, maltose, idose, malate, and chitin (Table 4).

In the $\mathrm{F}$ tick group, most upregulated unigenes on carbohydrate metabolism were glycosyl hydrolase (DN53826), mannose-binding lectin (DN36723), beta-galactosidase (DN53710), and $\alpha$-L-fucosidase (DN57768). Glycosyl hydrolase is a kind of enzyme that hydrolyzes glycosidic bonds, and plays an important role in the hydrolysis and synthesis of glycoconjugates and glycosidic compounds [70]. In invertebrate mannosebinding lectin was related to specific pattern recognition of the complement system via activation of the lectin pathway $[71,72]$. $\beta$-galactosidase is widely found in various animals, plants and microorganisms that hydrolyses the $\beta$-glycosidic bond formed between a galactose and its organic moiety $[20,63]$. The data on a digestive $\alpha-\mathrm{L}-$ fucosidase activity of Amblyomma cajennense Fabricius were described in a study and a plausible inference was present that the upregulation of the gene was probably related to removal of fucose produced by microorganisms in tick gut $[73,74]$. In our study, the $\alpha$-L-fucosidase was over-expressed both in the $\mathrm{F}$ tick and $\mathrm{H}$ tick group.

In the $\mathrm{H}$ tick group, most upregulated unigenes on carbohydrate metabolism were galectin (DN57588), glucose 6-phosphate dehydrogenase (DN56097) and 6-phosphofructo-2-kinase/fructose 2,6-bisphosphatase (DN57996). Galectins are a glycan-binding superfamily protein (lectins) that plays an important role in insect and tick development, interaction with pathogens, and the innate immune system by recognizing repeating saccharide units found on microbial surface glycoproteins in immunity, respectively $[75,76]$. Glucose 6-phosphate dehydrogenase (G6PDH) is a NADPH-producing enzyme that was of critical role in the oxidative stage of the pentose phosphate pathway [77]. A study on $R$. microplus G6PDH showed four transcripts differentially expressed in engorged and/or unfed adults in which G6PDH-D showed upregulation as the one found in this study [78]. The 6-phosphofructo-2-kinase/fructose 2, 6-bisphosphatase (PFK-2/FBase-2) is a bifunctional enzyme that catalyzes either the synthesis (PFK-2 activity) or the degradation (FBase-2 activity) of fructose-2, 6-bisphosphate [79]. The results revealed that upregulation of PFK-2/ FBase- 2 in $\mathrm{H}$ tick after long-term starvation might be related to fructose-2, 6-bisphosphate.

In the $M$ tick group, most of the unigenes related to carbohydrate as energy were not significantly upregulated, while unigenes on chitin synthesis and binding were significantly upregulated (Table 4). In the transcriptome of $D$. marginatus, up to 30 unigenes were matched with PF00379 domain and another 30 unigenes were matched with PF01607 in the Pfam database. These two chitin binding domains were generally found in arthropods [80]. The majority of them annotated as structural constituent of cuticle, peritrophic membrane chitin binding protein, and conserved hypothetical protein were upregulated in the $M$ tick group, and the highly expressed unigenes were cuticular proteins indicating that D. marginatus female adult shortly after completion of metamorphism were highly expressed, while the functional annotations of these proteins were inadequate. The chitin binding peritrophic-A domain (PF01607: CBM14) may be involved in formation and maintenance of peritrophic membrane [81] (Table 4, Additional file 5: Table S5). The peritrophic membrane is considered having the function of protecting microvilli of midgut epithelial cells from mechanical damage, pathogens and toxic substances, and acts as a semipermeable barrier allowing transport of small molecules and nutrients [82]. The structure of tick peritrophic membrane has been described in $I$. scapularis [83], I. ricinus [84], Haemaphysalis longicornis Neumann [85] and Ixodes dammini Spielman [86]. The chitin-binding cuticular protein (PF00379) includes an amino acid motif which functions to bind chitin, but the protein shows no sequence similarity to the known chitin-binding domain (PF01607: CBM14) found in chitinases and some peritrophic membrane proteins [80]. Cuticular protein comprises highly organized structural products that is formed as a layered and extracellularly secreted protein from the epidermis of insects [87]. The upregulated unigenes encoding cuticular proteins in newly molted ticks could be related to sclerotization and melanin formation of the tick surface cuticle, as a previous report has described at length that the two process may occur in concert in insects [88].

Ticks own effective defense mechanisms to protect from pathogenic microorganisms and to maintain the intestinal microbiota at a tolerable level [89]. In the $D$. marginatus transcriptome, 13 upregulated unigenes were among 27 unigenes annotated with immune functions putatively encoding defensins, lysozyme, alphamacroglobulin, and hemolectin (Table 5, Additional file 5: Table S5). Regarding defensins, there were upregulations shown in all three states of $D$. marginatus transcriptome data. The most highly expressed defensin was DN36307 (TPM: 2888.17) in the H tick group. Defen$\sin$ is an antibacterial peptide that is the major components of innate immunity in ticks and protect ticks from gram-negative, gram-positive bacteria, and plasmodium [90-92]. Lysozymes are effective peptides which play a role in carbohydrate digestion suppressing the proliferation of Gram-negative bacteria in the midgut [93]. Alpha-macroglobulin plays an important role in phagocytosis of microbes, and may specifically be involved in phagocytosis of a certain genus of microbes in ticks [94]. 
Table 4 Selected differentially expressed unigenes putatively involved in carbohydrate metabolism after blood-feeding and longterm starvation

\begin{tabular}{|c|c|c|c|c|c|c|}
\hline Protein/Function & Annotation & TPM F-tick & TPM H-tick & TPM M-tick & ANOVA ( $P$-value) & Unigene \\
\hline \multirow[t]{10}{*}{ Carbohydrate metabolism } & Glycosyl hydralase, sucrase-isomaltase, putative & 62.30 & 0.75 & 1.67 & 0 & DN53826 \\
\hline & Alpha-L-fucosidase, putative & 18.85 & 6.80 & 0.56 & 0 & DN57768 \\
\hline & Beta-N-acetylhexosaminidase, putative & 16.00 & 1.29 & 2.47 & 0 & DN48906 \\
\hline & Beta-glucuronidase-like isoform $X_{1}$ & 31.56 & 1.90 & 6.15 & 0 & DN55789 \\
\hline & Poly (ADP-ribose) glycohydrolase-like isoform X3 & 3.15 & 8.91 & 4.75 & 0.06 & DN48563 \\
\hline & Glucosidase II, putative & 39.01 & 23.50 & 5.26 & 0 & DN51547 \\
\hline & Glucose 6-phosphate dehydrogenase isoform C & 22.75 & 66.04 & 34.30 & 0.02 & DN56097 \\
\hline & Sucrase-isomaltase, intestinal-like & 0.21 & 2.16 & 8.15 & 0 & DN57119 \\
\hline & Alpha-L-iduronidase-like isoform X1 & 0.01 & 5.44 & 0.32 & 0 & DN34070 \\
\hline & $\begin{array}{l}\text { 6-phosphofructo-2-kinase/fructose 2,6-bisphos- } \\
\text { phatase, putative }\end{array}$ & 3.74 & 14.96 & 5.12 & 0.01 & DN57996 \\
\hline \multirow[t]{4}{*}{ Mannose metabolism } & Mannosyl-oligosaccharide glucosidase, putative & 45.53 & 14.34 & 5.08 & 0 & DN34438 \\
\hline & Phosphomannomutase, putative & 55.46 & 14.18 & 4.64 & 0 & DN49511 \\
\hline & Mannose-1-phosphate guanyltransferase beta-like & 99.60 & 39.29 & 10.32 & 0 & DN47789 \\
\hline & $\begin{array}{l}\text { Mannose-binding endoplasmic reticulum-golgi inter- } \\
\text { mediate compartment lectin }\end{array}$ & 160.77 & 41.80 & 8.51 & 0 & DN36723 \\
\hline \multirow[t]{4}{*}{ Galactose metabolism } & Glycosyl hydralase, sucrase-isomaltase, putative & 62.30 & 0.75 & 1.67 & 0 & DN53826 \\
\hline & Ixodes scapularis Beta-galactosidase precursor, putative & 37.86 & 5.82 & 0.40 & 0 & DN53710 \\
\hline & Beta-1,4-N-acetylgalactosaminyltransferase bre-4-like & 0.01 & 7.36 & 3.04 & 0 & DN52083 \\
\hline & Galectin, putative & 101.18 & $1,262.29$ & 317.33 & 0 & DN57588 \\
\hline \multirow[t]{5}{*}{ Carbohydrate transport } & UDP-galactose transporter, putative & 8.55 & 21.71 & 8.43 & 0.13 & DN52272 \\
\hline & Oxoglutarate/malate carrier protein, putative & 6.33 & 44.22 & 6.32 & 0 & DN57072 \\
\hline & Sodium-dependent glucose transporter, putative & 0.84 & 7.65 & 0.36 & 0 & DN43028 \\
\hline & $\begin{array}{l}\text { Solute carrier family } 2 \text {, facilitated glucose transporter } \\
\text { Member 10-like }\end{array}$ & 6.27 & 19.39 & 1.57 & 0 & DN56465 \\
\hline & Sugar transporter, putative & 1.99 & 12.64 & 1.20 & 0 & DN52986 \\
\hline \multirow[t]{11}{*}{ Chitin binding protein } & Cuticular protein, putative & 1.39 & 3.73 & $1,053.67$ & 0 & DN35205 \\
\hline & Cuticular protein, putative & 0.10 & 27.47 & 113.61 & 0 & DN57895 \\
\hline & Cuticular protein, putative & 0.14 & 27.03 & 380.74 & 0 & DN41881 \\
\hline & Peritrophin A, putative & 33.01 & 4.28 & 250.60 & 0 & DN52544 \\
\hline & Cuticular protein, putative & 1.41 & 74.29 & 4.48 & 0 & DN45546 \\
\hline & Hypothetical protein X975_15305 & 2.67 & 58.72 & 59.54 & 0 & DN35800 \\
\hline & Salivary mucin & 8.35 & 62.56 & 98.37 & 0 & DN48751 \\
\hline & Conserved hypothetical protein & 29.27 & 2.97 & 15.17 & 0 & DN55376 \\
\hline & Peritrophin A, putative & 43.35 & 2.96 & 17.71 & 0 & DN44759 \\
\hline & Peritrophin A, putative & 32.29 & 421.29 & 198.20 & 0 & DN57787 \\
\hline & Hypothetical protein & 130.84 & 26.98 & 80.95 & 0.03 & DN46721 \\
\hline \multirow[t]{9}{*}{ Cuticle protein } & Structural constituent of cuticle, putative & 0.04 & 0.09 & $1,571.25$ & 0 & DN43545 \\
\hline & Conserved hypothetical protein & 0.01 & 0.05 & 133.71 & 0 & DN54086 \\
\hline & Cuticle protein, putative & 125.92 & 3.26 & 255.44 & 0 & DN44745 \\
\hline & Conserved hypothetical protein & 1.78 & 0.23 & 230.19 & 0 & DN52842 \\
\hline & Peritrophic membrane chitin binding protein, putative & 32.02 & 0.00 & 0.15 & 0 & DN57209 \\
\hline & Calphotin-like & 0.03 & 17.80 & 424.74 & 0 & DN37939 \\
\hline & Structural constituent of cuticle, putative & 0.00 & 0.78 & 271.20 & 0 & DN35945 \\
\hline & Cuticle protein, putative & 166.79 & 0.00 & 0.39 & 0 & DN49371 \\
\hline & Cuticular protein, putative & 2.28 & 0.18 & 268.89 & 0 & DN55649 \\
\hline
\end{tabular}


Table 4 (continued)

\begin{tabular}{|c|c|c|c|c|c|c|}
\hline Protein/Function & Annotation & TPM F-tick & TPM H-tick & TPM M-tick & ANOVA ( $P$-value) & Unigene \\
\hline & Cuticle protein, putative & 0.01 & 0.11 & 104.18 & 0 & DN50628 \\
\hline & Cuticle protein, putative & $4,618.14$ & 3.97 & 5.64 & 0 & DN49371 \\
\hline & Conserved hypothetical protein & 1.78 & 0.23 & 230.19 & 0 & DN52842 \\
\hline & Cuticular protein & 0.87 & 391.58 & $1,332.75$ & 0 & DN49929 \\
\hline & Cuticle protein, putative & 0.79 & 153.69 & 230.33 & 0 & DN39452 \\
\hline & Cuticular protein, putative & 11.83 & 0.19 & 40.79 & 0 & DN51261 \\
\hline & Cuticle protein, putative & 125.92 & 3.26 & 255.44 & 0 & DN44745 \\
\hline & Cuticular protein, putative & 0.22 & 209.83 & 68.04 & 0 & DN55323 \\
\hline & Cuticular protein, putative & 2.36 & 2.31 & 39.73 & 0 & DN52617 \\
\hline & Cuticular protein-like protein & 7.12 & 2.89 & 55.46 & 0 & DN54351 \\
\hline & Cuticle protein & $3,195.63$ & 128.25 & $2,513.73$ & 0 & DN46667 \\
\hline
\end{tabular}

TPM values are the mean of the three biological replicates analyzed from each physiological condition. Significance analysis was based on one-way ANOVA $(P<0.05)$. Full information on unigene names, TPM data, and other information see Additional file 5: Table S5

Several $\alpha$-macroglobulins were upregulated in the $\mathrm{F}$ tick group after blood-feeding indicating the ticks were trying to control microbial populations in the system. Besides $\alpha$-macroglobulins, a hemolectin was upregulated in $\mathrm{F}$ tick group which is a major clotting factor in insects [95, 96], and also has a function help insect immune system against bacterial infection [97].

Ingestion of large amounts of host blood and the digestion of the blood meal increases redox pressure in ticks. Therefore, detoxifying mechanisms are required for counteracting redox pressure in ticks [98]. There were 64 upregulated transcripts coding for proteins with oxidoreductase activities, chaperone proteins, and proteins involved in iron and hemoglobin metabolism (Table 5). Most of the upregulated unigenes were in the $F$ tick group. In the differentially expressed unigenes, a glutathione S-transferase (GST) gene (DN56344) was highly expressed both in F tick (TPM: 2,285.92) and H tick (TPM: 1,869.90) groups. GSTs are known as genes of a superfamily that are involved in the detoxification of endogenous and xenobiotic compounds [99]. Bloodfeeding increased cellular stresses, and the majority of the GSTs were upregulated except two omega class GSTs (GSTO) that were upregulated in the $M$ tick group (DN51816; DN50592). The GSTOs were characterized in insects, and are over-express under stress response and at the presence of insecticides [100, 101]. Other highly expressed antioxidant proteins included catalase (DN57352; TPM: 624.90), protein disulfide isomerase (DN52494; TPM: 781.58) and glutathione peroxidase (DN40806; TPM: 1,434.27).
Heat-shock proteins (HSP) work as molecules involved in correction of protein folding which were over-expressed under heat shock and other stress responses as ticks ingesting host blood with elevated temperature, dealing with environmental stress, and neutralizing damage caused by toxic substances ingested with blood meal or produced during digestion of blood meal [102, 103]. We selected 16 differentially expressed HSPs including HSP90, HSP70, HSP60 and HSP20 (small HSPs) (Table 5). After blood-feeding, The HSP90 (DN49937; TPM: 312.01) over-expressed in the $\mathrm{F}$ tick group which was 5.8 folds the expression of the gene in the $\mathrm{H}$ tick group and 3 folds the expression of the gene in the $\mathrm{M}$ tick group. Notably a HSP20 (DN58874) was exclusively expressed in the $\mathrm{H}$ tick group. Two highly differentially expressed HSP70s (DN38789 and DN48195) were in the F tick group. Significantly upregulated and highly expressed HSP20s were also found in the $\mathrm{F}$ tick group implying upregulation of these genes may induced by feeding and bloodmeal digestion [46], while upregulation of unigenes including HSP60 (DN55315 and DN33794) and HSP20 (DN49326, DN46199 and DN44693) in the H tick group were likely caused by exhaustion of energy substance and environmental stress after long-term starvation $[104,105]$.

Ticks acquire iron and heme from host blood, while ticks can not utilize heme for bioavailable iron [106]. In the iron metabolism ticks would have to bear redox pressure from acquiring iron from host transferrin [28]. In the transcriptome of $D$. marginaus, two unigenes annotated 
Table 5 Selected differentially expressed unigenes putatively involved in defense response and antioxidant response after bloodfeeding and long-term starvation

\begin{tabular}{|c|c|c|c|c|c|c|}
\hline Protein/Function & Annotation & TPM F-tick & TPM H-tick & TPM M-tick & ANOVA ( $P$-value) & Unigene \\
\hline \multirow[t]{7}{*}{ Defensin } & Hebreain & 404.98 & 10.32 & 32.75 & 0 & DN38627 \\
\hline & Hebreain & 352.36 & $2,888.17$ & 618.80 & 0 & DN36307 \\
\hline & Antimicrobial peptide microplusin & 8.32 & 73.65 & 178.51 & 0 & DN42974 \\
\hline & Defensin & 21.66 & 14.95 & 124.77 & 0 & DN37748 \\
\hline & Putative defense protein 3-like protein & 76.62 & 8.28 & 2.10 & 0 & DN33990 \\
\hline & Putative defense protein 1 & 8.27 & 1.99 & 123.46 & 0 & DN44863 \\
\hline & Conserved hypothetical protein & 0.61 & 0.21 & 76.55 & 0 & DN51960 \\
\hline \multirow[t]{2}{*}{ Alpha-macroglobulin } & Alpha-macroglobulin, putative & 80.00 & 9.26 & 5.26 & 0 & DN48632 \\
\hline & Alpha-macroglobulin, putative & 28.02 & 0.98 & 3.19 & 0 & DN55988 \\
\hline \multirow[t]{3}{*}{ Lysozyme } & Lysozyme & 30.22 & 104.76 & 10.41 & 0 & DN43837 \\
\hline & Lysozyme, putative & 24.63 & 18.74 & 51.02 & 0.01 & DN54894 \\
\hline & Gamma-interferon inducible lysosomal thiol reductase & 1.89 & 25.10 & 1.06 & 0 & DN37451 \\
\hline Hemolectin & Hemolectin, putative & 9.64 & 1.61 & 0.20 & 0 & DN58201 \\
\hline \multirow[t]{16}{*}{ Glutathion S-transferase } & Putative glutathione S-transferase & 54.51 & 4.40 & 0.45 & 0 & DN41472 \\
\hline & Glutathione S-transferase & 377.46 & 61.22 & 21.56 & 0 & DN45728 \\
\hline & Glutathione S-transferase, putative & 76.98 & 12.08 & 12.37 & 0 & DN49881 \\
\hline & Glutathione S-transferase, putative & 63.43 & 9.89 & 9.71 & 0 & DN40498 \\
\hline & Putative glutathione S-transferase & 103.27 & 2.50 & 0.21 & 0 & DN29689 \\
\hline & Glutathione S-transferase, putative & 141.94 & 17.66 & 43.40 & 0 & DN39983 \\
\hline & Glutathione S-transferase, putative & 83.97 & 50.83 & 18.84 & 0 & DN51596 \\
\hline & Glutathione S-transferase, putative & 34.12 & 23.17 & 3.01 & 0 & DN46189 \\
\hline & Glutathione S-transferase, putative & 24.53 & 3.86 & 15.36 & 0 & DN48615 \\
\hline & Glutathione S-transferase, putative & 4.67 & 2.92 & 11.30 & 0 & DN51816 \\
\hline & Glutathione S-transferase & 38.31 & 40.50 & 80.58 & 0 & DN50592 \\
\hline & Putative glutathione S-transferase & 145.21 & 23.61 & 6.79 & 0.01 & DN38169 \\
\hline & Putative glutathione S-transferase & $2,285.92$ & $1,869.90$ & 234.28 & 0.01 & DN56344 \\
\hline & Glutathione S-transferase & 17.36 & 2.77 & 11.28 & 0.01 & DN50142 \\
\hline & Glutathione S-transferase, putative & 70.00 & 33.69 & 22.33 & 0.01 & DN44573 \\
\hline & Microsomal glutathione S-transferase & 44.68 & 67.61 & 24.03 & 0.03 & DN40419 \\
\hline \multirow[t]{8}{*}{ Antioxidant activity } & Catalase & 624.90 & 207.05 & 171.21 & 0 & DN57352 \\
\hline & Protein disulfide isomerase- 2 & 408.68 & 45.01 & 22.40 & 0 & DN50839 \\
\hline & Protein disulfide isomerase & 781.58 & 202.00 & 98.63 & 0 & DN52494 \\
\hline & Thiol-disulfide isomerase, putative & 41.52 & 14.07 & 2.77 & 0 & DN46036 \\
\hline & Protein disulfide isomerase-1 & 95.20 & 34.10 & 14.70 & 0 & DN50839 \\
\hline & Thioredoxin peroxidase & 186.76 & 29.83 & 10.68 & 0 & DN42520 \\
\hline & $16 \mathrm{kDa}$ thioredoxion, putative & 155.43 & 66.01 & 29.23 & 0 & DN37149 \\
\hline & Glutathione peroxidase & $1,434.27$ & 174.50 & 46.00 & 0 & DN40806 \\
\hline \multirow[t]{2}{*}{ Iron metabolism } & Ferritin 2 & 78.53 & 84.06 & 22.75 & 0.01 & DN51538 \\
\hline & Transferrin receptor, putative & 80.18 & 0.03 & 0.03 & 0 & DN54992 \\
\hline \multirow[t]{4}{*}{ Superoxide dismutase } & Hypothetical protein & 16.17 & 0.75 & 0.49 & 0 & DN50617 \\
\hline & Superoxide dismutase $\mathrm{Cu}-\mathrm{Zn}$, putative & 17.30 & 5.66 & 7.58 & 0 & DN52749 \\
\hline & Unnamed protein product & 22.05 & 38.38 & 96.10 & 0 & DN40853 \\
\hline & Superoxide dismutase [Cu-Zn] isoform X2 & 10.76 & 2.49 & 6.86 & 0 & DN41589 \\
\hline \multirow[t]{4}{*}{ Heat shock proteins } & Heat shock proteins 90 , putative & 312.01 & 72.53 & 29.59 & 0 & DN49937 \\
\hline & Heat shock 70 kDa protein & 287.74 & 81.31 & 31.94 & 0 & DN38789 \\
\hline & Heat shock 70 kDa protein & 341.26 & 225.29 & 70.48 & 0 & DN48195 \\
\hline & Heat shock protein 70 & 1.54 & 10.26 & 9.86 & 0 & DN48944 \\
\hline
\end{tabular}


Table 5 (continued)

\begin{tabular}{|c|c|c|c|c|c|c|}
\hline Protein/Function & Annotation & TPM F-tick & TPM H-tick & TPM M-tick & ANOVA ( $P$-value) & Unigene \\
\hline & Heat shock proteins 60 & 51.74 & 106.17 & 19.96 & 0 & DN55315 \\
\hline & Mitochondrial chaperonin hsp60 & 2.19 & 18.04 & 0.13 & 0 & DN33794 \\
\hline & Heat shock HSP20 protein, putative & 5.00 & 40.29 & 7.56 & 0 & DN44693 \\
\hline & Putative heat shock-related protein & 98.50 & 1.11 & 2.96 & 0 & DN43840 \\
\hline & Stress-induced protein 1 & 0.00 & 17.55 & 0.00 & 0 & DN58874 \\
\hline & PREDICTED: heat shock protein 16-like & 0.75 & 19.81 & 0.14 & 0 & DN37163 \\
\hline & Small heat shock protein I & 829.95 & 124.30 & 124.53 & 0 & DN45806 \\
\hline & Small heat shock protein I & 860.03 & 112.86 & 55.58 & 0 & DN46736 \\
\hline & Putative heat shock-related protein & 79.00 & 6.59 & 6.37 & 0 & DN45729 \\
\hline & Small heat shock protein II & 13.34 & 61.93 & 28.28 & 0 & DN46199 \\
\hline & Heat shock protein, putative & 92.50 & 174.46 & 24.35 & 0 & DN49326 \\
\hline
\end{tabular}

TPM values are the mean of the three biological replicates analyzed from each physiological condition. Significance analysis was based on one-way ANOVA $(P<0.05)$. Full information on unigene names, TPM data, and other information see Additional file 5: Table S5

on iron metabolism were significantly upregulated in the $\mathrm{F}$ tick group including ferritin 2 (DN51538) and transferrin receptor (DN54992) (Table 5). Tick ferritin 2 is a unique secreted protein that is conserved in ticks [30], and tick ferritin 2 is a gut-specific protein which is secreted into the hemolymph transporting bioavailable iron in between organs [107]. The iron in host transferrin was released by lysosome in tick midgut through endocytosis that was triggered when transferrin receptor conjugated with host transferrin [106, 108], but further detailed research is needed to characterize molecular function of tick transferrin receptors. Generally, feeding and digestion of haemoglobin in blood meal releases large amounts of heme and subsequently cause the upregulation of antioxidant genes, whereas exhaustion of energy reserve would increase reactive oxygen species, which in turn would exacerbate starvation-induced autophagy [109].

\section{RT-qPCR validation of RNA-seq data}

In order to validate RNA-seq results, the expression level of six selected upregulated genes were assessed by RTqPCR, using $e f-1 \alpha$ (DN43634) as a reference. The selected genes encoded the following proteins: ferritin 1; ferritin 2; HSP70; galectin; GST; and Dm86 (Fig. 7). For checking the suitability of the primer pairs, the amplification products were electrophoresed in an agarose gel, and the density of the bands of expected size were measured for all genes, including the housekeeping genes. The comparison between the RT-qPCR and RNA-seq results confirmed that all genes showed a similar trend in transcriptional expression changes, the normalized fold change values of all unigenes by ef-1 $\alpha$ (DN43634) were recorded in Additional file 5: Table S5. Transcripts encoding ferritin 1, HSP70, GST, galectin and Dm86 showed rather similar expression patterns, while, ferritin 2 did not show differential expression in RT-qPCR (Fig. 7). The less consistent result obtained from RT-qPCR and RNA-seq may be due to several reasons including variability in the expression of the reference gene in different biological samples or differences in data standardization method between RT-qPCR and RNA-Seq analyses [37].

The biological significance of the gene expression profiles is important, but the gene expression alone could not characterize them, as further investigations are required. In total, the differences among the gene expression profiles indicate that the transcriptome of $D$. marginatus female ticks are informative, which may provide data for further research on this tick species.

\section{Conclusions}

In this study we expanded sequence information of $D$. marginatus by RNA-seq data and provided functional annotations of the genes of this tick species on a wholebody transcriptome level. We found that blood-feeding and starvation induced a strong upregulation of unigenes associated with proteins, lipids and carbohydrates metabolism, as well as unigenes associated with defense mechanism and antioxidant activity. Overall, these results would facilitate the understanding of $D$. marginatus genes encoding potential target molecules for efficient anti-tick interventions. 


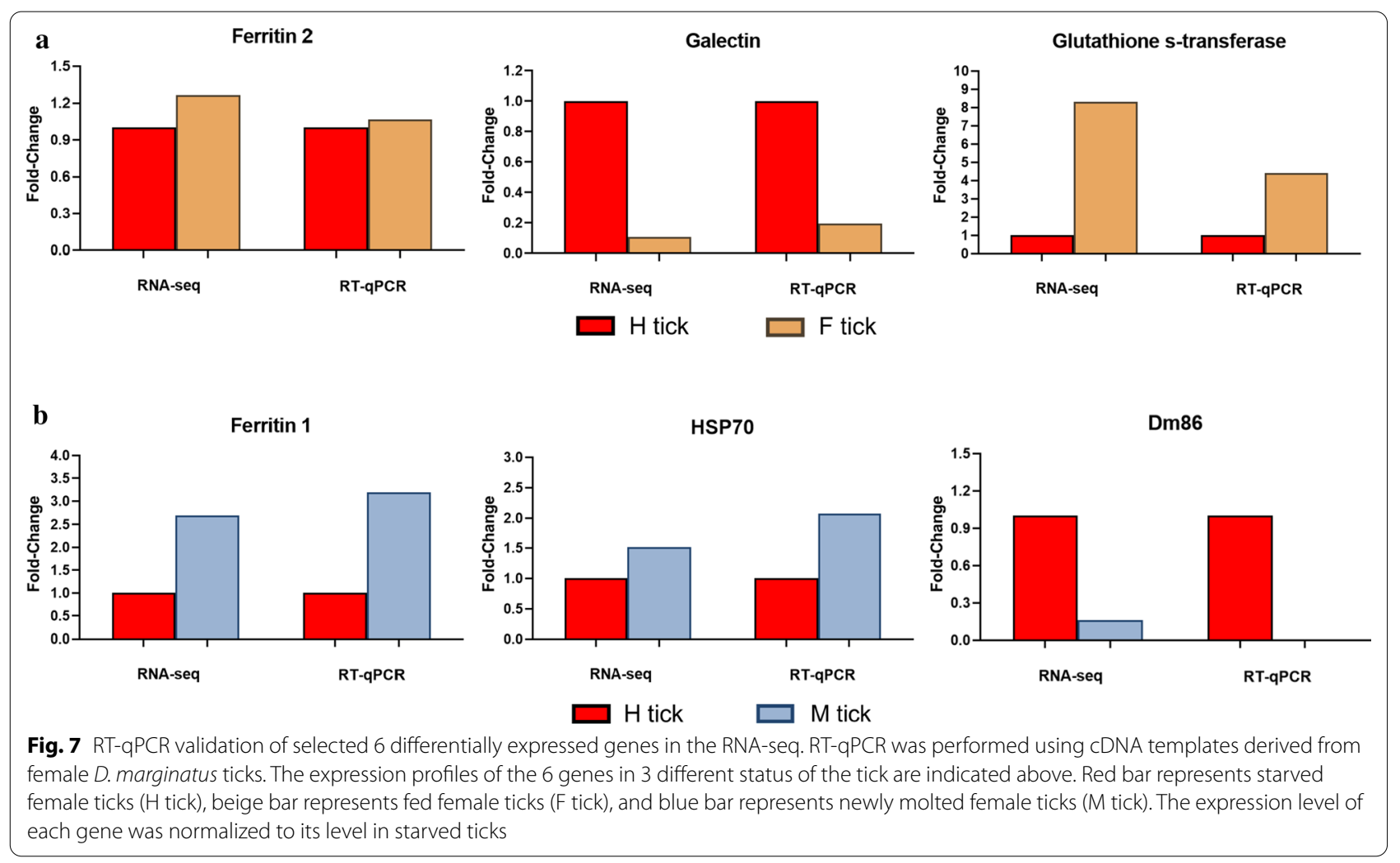

\section{Supplementary information}

Supplementary information accompanies this paper at https://doi. org/10.1186/s13071-020-04442-2.

Additional file 1: Table S1. Specific primers used in the RT-qPCR validation of RNA-seq results.

Additional file 2: Table S2. Overview of sequencing quality control.

Additional file 3: Table S3. Annotation summary with DIAMOND software.

Additional file 4: Table S4. Raw reads alignment results.

Additional file 5: Table S5. Annotation table of D. marginatus female adult transcriptome analysis.

Additional file 6: Table S6. Unigenes used in molecular function, cellular component and biological process anaylisis by GO annotation.

Additional file 7: Figure S1. Genes differentially expressed between groups after blood-feeding and long-term starvation.

\section{Abbreviations}

RNA-seq: RNA sequencing; TPM: transcripts per million; DEGs: differentially expressed genes; RT-qPCR: real-time quantitative PCR; GEO: Gene Expression Omnibus database; GO: Gene Ontology database; KEGG: Kyoto Encyclopedia of Genes and Genomes database.

\section{Acknowledgements}

We thank three anonymous reviewers for their constructive comments on the paper and Ms Wulijian and Mr Sadilamu from our laboratory for caring and performing health examinations to the experimental horse regularly.

\section{Authors' contributions}

$\mathrm{CB}$ and $\mathrm{QG}$ conceived the study. EH and YM designed the experiments. EH, YM, ML and HY collected the data. EH, YM and ZH analyzed the data. EH wrote the original manuscript. All the authors contributed substantially to revision of the manuscript and take full responsibility for the content of the paper. All authors read and approved the final manuscript.

\section{Funding}

This study was supported by a grant from the National Natural Science Foundation of China (project number 31660711) to CB, and by a grant from the Educational Commission of Xinjiang Autonomous Region of China (project number XJEDU20201008) to QG.

\section{Availability of data and materials}

The datasets supporting the conclusions of this article are available in the GEO database of GenBank repository (https://www.ncbi.nlm.nih.gov/geo/query/ acc.cgi?acc=GSE151667) with accession number GSE151667.

\section{Ethics approval and consent to participate}

Experimental animals were kept in veterinary facilities under natural condition. All animals involved in this experiment and the related experimental procedures were conducted under the supervision of the Animal Welfare Committee of Xinjiang Agricultural University, permission number 2019006, and the authors approved to participate.

\section{Consent for publication}

Not applicable.

\section{Competing interests}

The authors declare that they have no competing interests. 


\begin{abstract}
Author details
${ }^{1}$ College of Animal Science, Xinjiang Agricultural University, Ürümqi 830052, Xinjiang Uygur Autonomous Region, People's Republic of China. ${ }^{2}$ College of Veterinary Medicine, Xinjiang Agricultural University, Ürümqi 830052, Xinjiang Uygur Autonomous Region, People's Republic of China. ${ }^{3} \mathrm{Col}-$ lege of Animal Science and Technology, Qingdao Agricultural University, Qingdao 266109, Shandong Province, People's Republic of China. ${ }^{4}$ National Research Center for Protozoan Diseases, Obihiro University of Agriculture and Veterinary Medicine, Obihiro, Hokkaido 080-8555, Japan. ${ }^{5}$ Bayingol Vocational and Technical College, Korla 841000, Xinjiang Uygur Autonomous Region, People's Republic of China.
\end{abstract}

Received: 13 August 2020 Accepted: 30 October 2020

Published online: 10 November 2020

\section{References}

1. de la Fuente J. Controlling ticks and tick-borne diseases... looking forward. Ticks Tick Borne Dis. 2018;9:1354-7.

2. Hilpertshauser H, Deplazes P, Schnyder M, Gern L, Mathis A. Babesia spp. identified by PCR in ticks collected from domestic and wild ruminants in southern Switzerland. Appl Environ Microbiol. 2006;72:6503-7.

3. Chisu V, Alberti A, Zobba R, Foxi C, Masala G. Molecular characterization and phylogenetic analysis of Babesia and Theileria spp. in ticks from domestic and wild hosts in Sardinia. Acta Trop. 2019;196:60-5.

4. Song R, Wang Q, Guo F, Liu X, Song S, Chen C, et al. Detection of Babesia spp., Theileria spp. and Anaplasma ovis in Border Regions, northwestern China. Transbound Emerg Dis. 2018;65:1537-44.

5. Li J, Li Y, Moumouni PFA, Lee SH, Galon EM, Tumwebaze MA, et al. First description of Coxiella burnetii and Rickettsia spp. infection and molecular detection of piroplasma co-infecting horses in Xinjiang Uygur Autonomous Region. China. Parasitol Int. 2019;76:102028.

6. Bonnet S, de la Fuente J, Nicollet P, Liu X, Madani N, Blanchard B, et al. Prevalence of tick-borne pathogens in adult Dermacentor spp. ticks from nine collection sites in France. Vector Borne Zoonotic Dis. 2013;13:226-36.

7. Wang Q, Zhao S, Wureli H, Xie S, Chen C, Wei Q, et al. Brucella melitensis and $B$. abortus in eggs, larvae and engorged females of Dermacentor marginatus. Ticks Tick Borne Dis. 2018;9:1045-8.

8. Chrudimska T, Cerovsky V, Slaninova J, Rego RO, Grubhoffer L. Defensin from the ornate sheep tick Dermacentor marginatus and its effect on Lyme borreliosis spirochetes. Dev Comp Immunol. 2014;46:165-70.

9. Liu X, Zhang X, Wang Z, Dong Z, Xie S, Jiang M, et al. A tentative tamdy orthonairovirus related to febrile illness in Northwestern China. Clin Infect Dis. 2019;70:2155-60.

10. de la Fuente J, Estrada-Pena A. Why new vaccines for the control of ectoparasite vectors have not been registered and commercialized? Vaccines (Basel). 2019;7:75.

11. Benelli G, Maggi F, Romano D, Stefanini C, Vaseeharan B, Kumar S, et al. Nanoparticles as effective acaricides against ticks-A review. Ticks Tick Borne Dis. 2017;8:821-6.

12. Vudriko P, Okwee-Acai J, Byaruhanga J, Tayebwa DS, Omara R, Muhindo $\mathrm{JB}$, et al. Evidence-based tick acaricide resistance intervention strategy in Uganda: concept and feedback of farmers and stakeholders. Ticks Tick Borne Dis. 2018;9:254-65

13. de la Fuente J, Kopáček P, Lew-Tabor A, Maritz-Olivier C. Strategies for new and improved vaccines against ticks and tick-borne diseases. Parasite Immunol. 2016;38:754-69.

14. Diaz-Sanchez S, Estrada-Pena A, Cabezas-Cruz A, de la Fuente J. Evolutionary insights into the tick hologenome. Trends Parasitol. 2019;35:725-37

15. Gulia-Nuss M, Nuss AB, Meyer JM, Sonenshine DE, Roe RM, Waterhouse RM, et al. Genomic insights into the Ixodes scapularis tick vector of Lyme disease. Nat Commun. 2016;7:10507.

16. Jia N, Wang J, Shi W, Du L, Sun Y, Zhan W, et al. Large-scale comparative analyses of tick genomes elucidate their genetic diversity and vector capacities. Cell. 2020;182:1328-40.

17. Charrier NP, Couton M, Voordouw MJ, Rais O, Durand-Hermouet A, Hervet $C$, et al. Whole body transcriptomes and new insights into the biology of the tick Ixodes ricinus. Parasit Vectors. 2018;11:364.
18. Rosendale AJ, Dunlevy ME, McCue MD, Benoit JB. Progressive behavioural, physiological and transcriptomic shifts over the course of prolonged starvation in ticks. Mol Ecol. 2019;28:49-65.

19. Hao J, Luo J, Chen Z, Ren Q, Guo J, Liu X, et al. MicroRNA-275 and its target Vitellogenin-2 are crucial in ovary development and blood digestion of Haemaphysalis longicornis. Parasit Vectors. 2017;10:253.

20. Cabezas-Cruz A, Alberdi P, Valdes JJ, Villar M, de la Fuente J. Anaplasma phagocytophilum Infection subverts carbohydrate metabolic pathways in the tick vector, Ixodes scapularis. Front Cell Infect Microbiol. 2017;7:23.

21. Charrier NP, Hermouet A, Hervet C, Agoulon A, Barker SC, Heylen D, et al. A transcriptome-based phylogenetic study of hard ticks (Ixodidae). Sci Rep. 2019;9:12923.

22. Perner J, Provazník J, Schrenková J, Urbanová V, Ribeiro JM, Kopáček P. RNA-seq analyses of the midgut from blood- and serum-fed Ixodes ricinus ticks. Sci Rep. 2016;6:36695.

23. Contreras M, Villar M, de la Fuente J. A Vaccinomics approach for the identification of tick protective antigens for the control of Ixodes ricinus and Dermacentor reticulatus infestations in companion animals. Front Physiol. 2019;10:977.

24. de la Fuente J, Merino O. Vaccinomics, the new road to tick vaccines. Vaccine. 2013;31:5923-9.

25. Rand KN, Moore T, Sriskantha A, Spring K, Tellam R, Willadsen P, et al. Cloning and expression of a protective antigen from the cattle tick Boophilus microplus. Proc Natl Acad Sci USA. 1989;86:9657-61.

26. Rodríguez-Valle M, Taoufik A, Valdés M, Montero C, Ibrahin H, Hassan SM, et al. Efficacy of Rhipicephalus (Boophilus) microplus Bm86 against Hyalomma dromedarii and Amblyomma cajennense tick infestations in camels and cattle. Vaccine. 2012;30:3453-8.

27. Franta Z, Frantová H, Konvičková J, Horn M, Sojka D, Mareš M, et al. Dynamics of digestive proteolytic system during blood feeding of the hard tick Ixodes ricinus. Parasit Vectors. 2010;3:119.

28. Perner J, Sobotka R, Sima R, Konvickova J, Sojka D, Oliveira PL, et al. Acquisition of exogenous haem is essential for tick reproduction. Elife. 2016;5:e12318.

29. Sojka D, Franta Z, Horn M, Caffrey CR, Mareš M, Kopáček P. New insights into the machinery of blood digestion by ticks. Trends Parasitol. 2013;29:276-85.

30. Hajdusek O, Almazan C, Loosova G, Villar M, Canales M, Grubhoffer L, et al. Characterization of ferritin 2 for the control of tick infestations. Vaccine. 2010;28:2993-8.

31. Contreras M, de la Fuente J. Control of infestations by Ixodes ricinus tick larvae in rabbits vaccinated with aquaporin recombinant antigens. Vaccine. 2017;35:1323-8.

32. Mitchell RD, Sonenshine DE, Pérez de León AA. Vitellogenin receptor as a target for tick control: a mini-review. Front Physiol. 2019;10:618.

33. Benelli G, Pavela R, Canale A, Mehlhorn H. Tick repellents and acaricides of botanical origin: a green roadmap to control tick-borne diseases? Parasitol Res. 2016;115:2545-60.

34. Xiong C, Kaczmarek K, Zabrocki J, Nachman RJ, Pietrantonio PV. Activity of native tick kinins and peptidomimetics on the cognate target $G$ protein-coupled receptor from the cattle fever tick, Rhipicephalus microplus (Acari: Ixodidae). Pest Manag Sci. 2019;10:1008.

35. Magdas C, Magdas VA, Mihalca AD, Baciu H, Gherman CM, Stefanut CL, et al. Laboratory development of Dermacentor marginatus ticks (Acari: Ixodidae) at two temperatures. Exp Appl Acarol. 2015;67:309-15.

36. Bensaoud C, Nishiyama MY Jr, Ben Hamda C, Lichtenstein F, Castro de Oliveira U, Faria F, et al. De novo assembly and annotation of Hyalomma dromedarii tick (Acari: Ixodidae) sialotranscriptome with regard to gender differences in gene expression. Parasit Vectors. 2018;11:314.

37. Xu XL, Cheng TY, Yang H, Liao ZH. De novo assembly and analysis of midgut transcriptome of Haemaphysalis flava and identification of genes involved in blood digestion, feeding and defending from pathogens. Infect Genet Evol. 2016;38:62-72.

38. Kechin A, Boyarskikh U, Kel A, Filipenko M. cutPrimers: a new tool for accurate cutting of primers from reads of targeted next generation sequencing. J Comput Biol. 2017;24:1138-43.

39. Grabherr MG, Haas BJ, Yassour M, Levin JZ, Thompson DA, Amit I, et al. Full-length transcriptome assembly from RNA-seq data without a reference genome. Nat Biotechnol. 2011;29:644-52.

40. Haas BJ, Papanicolaou A, Yassour M, Grabherr M, Blood PD, Bowden J, et al. De novo transcript sequence reconstruction from RNA-seq using 
the Trinity platform for reference generation and analysis. Nat Protoc. 2013;8:1494-512.

41. Buchfink B, Xie C, Huson DH. Fast and sensitive protein alignment using DIAMOND. Nat Methods. 2015;12:59-60.

42. Patro R, Duggal G, Love MI, Irizarry RA, Kingsford C. Salmon provides fast and bias-aware quantification of transcript expression. Nat Methods. 2017;14:417-9.

43. Mortazavi A, Williams BA, McCue K, Schaeffer L, Wold B. Mapping and quantifying mammalian transcriptomes by RNA-seq. Nat Methods. 2008:5:621-8

44. Robinson MD, McCarthy DJ, Smyth GK. edgeR: a Bioconductor package for differential expression analysis of digital gene expression data. Bioinformatics. 2010;26:139-40.

45. Schmittgen TD, Livak KJ. Analyzing real-time PCR data by the comparative C(T) method. Nat Protoc. 2008;3:1101-8.

46. Oleaga A, Obolo-Mvoulouga P, Manzano-Román R, Pérez-Sánchez R. Functional annotation and analysis of the Ornithodoros moubata midgut genes differentially expressed after blood feeding. Ticks Tick Borne Dis. 2017;8:693-708.

47. Guo J, Sun Y, Luo X, Li M, He P, He L, et al. De novo transcriptome sequencing and comparative analysis of Haemaphysalis flava Neumann, 1897 at larvae and nymph stages. Infect Genet Evol. 2019;75:104008,

48. De Marco L, Epis S, Comandatore F, Porretta D, Cafarchia C, Mastrantonio $\mathrm{V}$, et al. Transcriptome of larvae representing the Rhipicephalus sanguineus complex. Mol Cell Probes. 2017;31:85-90.

49. Richards SA, Stutzer C, Bosman AM, Maritz-Olivier C. Transmembrane proteins - mining the cattle tick transcriptome. Ticks Tick Borne Dis. 2015;6:695-710.

50. Smith AD, Kaufman WR. Molecular characterization of two vitellogenin genes from the tick, Amblyomma hebraeum (Acari: Ixodidae). Ticks Tick Borne Dis. 2014;5:821-33.

51. Lara FA, Lins U, Paiva-Silva G, Almeida IC, Braga CM, Miguens FC, et al. A new intracellular pathway of haem detoxification in the midgut of the cattle tick Boophilus microplus: aggregation inside a specialized organelle, the hemosome. J Exp Biol. 2003;206:1707-15.

52. Lara FA, Lins U, Bechara GH, Oliveira PL. Tracing heme in a living cell: hemoglobin degradation and heme traffic in digest cells of the cattle tick Boophilus microplus. J Exp Biol. 2005;208:3093-101.

53. Umemiya-Shirafuji R, Matsuo T, Liao M, Boldbaatar D, Battur B, Suzuki $H$, et al. Increased expression of ATG genes during nonfeeding periods in the tick Haemaphysalis longicornis. Autophagy. 2010;6:473-81.

54. Moura-Martiniano NO, Machado-Ferreira E, Gazêta GS, Soares CAG. Relative transcription of autophagy-related genes in Amblyomma sculptum and Rhipicephalus microplus ticks. Exp Appl Acarol. 2017;73:401-28.

55. Umemiya-Shirafuji R, Galay RL, Maeda H, Kawano S, Tanaka T, Fukumoto $\mathrm{S}$, et al. Expression analysis of autophagy-related genes in the hard tick Haemaphysalis longicornis. Vet Parasitol. 2014;201:169-75.

56. Canavoso LE, Jouni ZE, Karnas KJ, Pennington JE, Wells MA. Fat metabolism in insects. Annu Rev Nutr. 2001;21:23-46.

57. Arrese EL, Soulages JL. Insect fat body: energy, metabolism, and regulation. Annu Rev Entomol. 2010;55:207-25.

58. Thompson DM, Khalil SMS, Jeffers LA, Sonenshine DE, Mitchell RD, Osgood CJ, et al. Sequence and the developmental and tissue-specific regulation of the first complete vitellogenin messenger RNA from ticks responsible for heme sequestration. Insect Biochem Mol Biol. 2007;37:363-74

59. Donohue KV, Khalil SMS, Mitchell RD, Sonenshine DE, Roe RM. Molecular characterization of the major hemelipoglycoprotein in Ixodid ticks. Insect Mol Biol. 2008;17:197-208.

60. Khalil SMS, Donohue KV, Thompson DM, Jeffers LA, Ananthapadmanaban U, Sonenshine DE, et al. Full-length sequence, regulation and developmental studies of a second vitellogenin gene from the American dog tick, Dermacentor variabilis. J Insect Physiol. 2011;57:400-8.

61. Brown MS, Goldstein JL. A receptor-mediated pathway for cholesterol homeostasis. Science. 1986;232:34-47.

62. Seixas A, Alzugaray MF, Tirloni L, Parizi LF, Pinto AFM, Githaka NW, et al. Expression profile of Rhipicephalus microplus vitellogenin receptor during oogenesis. Ticks Tick Borne Dis. 2018;9:72-81.

63. Oleaga A, Obolo-Mvoulouga P, Manzano-Roman R, Perez-Sanchez R. De novo assembly and analysis of midgut transcriptome of the argasid tick Ornithodoros erraticus and identification of genes differentially expressed after blood feeding. Ticks Tick Borne Dis. 2018;9:1537-54.

64. Li C, Wang M, Zhang T, He Q, Shi H, Luo J, et al. Insulin-induced gene 1 and 2 isoforms synergistically regulate triacylglycerol accumulation, lipid droplet formation, and lipogenic gene expression in goat mammary epithelial cells. J Dairy Sci. 2019;102:1736-46.

65. Gilbert LI, Chino H. Transport of lipids in insects. J Lipid Res. 1974;15:439-56.

66. Kluck GEG, Silva Cardoso L, De Cicco NNT, Lima MS, Folly E, Atella GC. A new lipid carrier protein in the cattle tick Rhipicephalus microplus. Ticks Tick Borne Dis. 2018;9:850-9.

67. Vanier MT. Niemann-Pick disease type C. Orphanet J Rare Dis. 2010;5:16.

68. Hannun YA, Obeid LM. Sphingolipids and their metabolism in physiology and disease. Nat Rev Mol Cell Biol. 2017;19:175-91.

69. Anderson JM, Sonenshine DE, Valenzuela JG. Exploring the mialome of ticks: an annotated catalogue of midgut transcripts from the hard tick, Dermacentor variabilis (Acari: Ixodidae). BMC Genomics. 2008;9:552.

70. Abdul Manas NH, Md Illias R, Mahadi NM. Strategy in manipulating transglycosylation activity of glycosyl hydrolase for oligosaccharide production. Crit Rev Biotechnol. 2018;38:272-93.

71. Ma YG, Cho MY, Zhao M, Park JW, Matsushita M, Fujita T, et al. Human mannose-binding lectin and L-ficolin function as specific pattern recognition proteins in the lectin activation pathway of complement. J Biol Chem. 2004;279:25307-12.

72. Coumou J, Wagemakers A, Narasimhan S, Schuijt TJ, Ersoz JI, Oei A, et al. The role of mannose binding lectin in the immune response against Borrelia burgdorferi sensu lato. Sci Rep. 2019;9:1431.

73. Moreti R, Perrella NN, Lopes AR. Carbohydrate digestion in ticks and a digestive alpha-L-fucosidase. J Insect Physiol. 2013;59:1069-75.

74. Chessa D, Winter MG, Jakomin M, Baumler AJ. Salmonella enterica serotype Typhimurium Std fimbriae bind terminal alpha $(1,2)$ fucose residues in the cecal mucosa. Mol Microbiol. 2009;71:864-75.

75. Huang X, Tsuji N, Miyoshi T, Nakamura-Tsuruta S, Hirabayashi J, Fujisaki K. Molecular characterization and oligosaccharide-binding properties of a galectin from the argasid tick Ornithodoros moubata. Glycobiology. 2007;17:313-23.

76. Pace KE, Baum LG. Insect galectins: roles in immunity and development Glycoconj J. 2002;19:607-14.

77. Stanton RC. Glucose-6-phosphate dehydrogenase, NADPH, and cell survival. IUBMB Life. 2012;64:362-9.

78. Olafson PU, Temeyer KB, Pruett JH. Multiple transcripts encode glucose 6-phosphate dehydrogenase in the southern cattle tick, Rhipicephalus (Boophilus) microplus. Exp Appl Acarol. 2011;53:147-65.

79. Baillet A, Hograindleur MA, El Benna J, Grichine A, Berthier S, Morel $F$, et al. Unexpected function of the phagocyte NADPH oxidase in supporting hyperglycolysis in stimulated neutrophils: key role of 6-phosphofructo-2-kinase. FASEB J. 2017;31:663-73.

80. Rebers JE, Willis JH. A conserved domain in arthropod cuticular proteins binds chitin. Insect Biochem Mol Biol. 2001;31:1083-93.

81. Elvin CM, Vuocolo T, Pearson RD, East IJ, Riding GA, Eisemann CH, et al. Characterization of a major peritrophic membrane protein, peritrophin-44, from the larvae of Lucilia cuprina. cDNA and deduced amino acid sequences. J Biol Chem. 1996;271:8925-35.

82. Lehane MJ. Peritrophic matrix structure and function. Annu Rev Entomol. 1997:42:525-50.

83. Kariu T, Smith A, Yang X, Pal U. A chitin deacetylase-like protein is a predominant constituent of tick peritrophic membrane that influences the persistence of Lyme disease pathogens within the vector. PLoS ONE. 2013;8:e78376.

84. Zhu Z, Gern L, Aeschlimann A. The peritrophic membrane of Ixodes ricinus. Parasitol Res. 1991;77:635-41.

85. Matsuo T, Sato M, Inoue N, Yokoyama N, Taylor D, Fujisaki K. Morphological studies on the extracellular structure of the midgut of a tick, Haemaphysalis longicornis (Acari: Ixodidae). Parasitol Res. 2003:90:243-8.

86. Rudzinska MA, Spielman A, Lewengrub S, Piesman J, Karakashian S. Penetration of the peritrophic membrane of the tick by Babesia microti. Cell Tissue Res. 1982;221:471-81.

87. Andersen SO, Højrup P, Roepstorff P. Insect cuticular proteins. Insect Biochem Mol Biol. 1995;25:153-76.

88. Andersen SO. Insect cuticular sclerotization: a review. Insect Biochem Mol Biol. 2010:40:166-78. 
89. Bonnet SI, Binetruy F, Hernández-Jarguín AM, Duron O. The tick microbiome: why $\mathrm{N}=$ non-pathogenic microorganisms matter in tick biology and pathogen transmission. Front Cell Infect Microbiol. 2017;7:236.

90. Couto J, Tonk M, Ferrolho J, Antunes S, Vilcinskas A, de la Fuente J, et al. Antiplasmodial activity of tick defensins in a mouse model of malaria. Ticks Tick Borne Dis. 2018;9:844-9.

91. Wang J, Bian G, Pan W, Feng T, Dai J. Molecular characterization of a defensin gene from a hard tick, Dermacentor silvarum. Parasit Vectors. 2015:8:25.

92. Chrudimská T, Čeřovský V, Slaninová J, Rego ROM, Grubhoffer L. Defensin from the ornate sheep tick Dermacentor marginatus and its effect on Lyme borreliosis spirochetes. Dev Comp Immunol. 2014;46:165-70.

93. Tanaka T, Kawano S, Nakao S, Umemiya-Shirafuji R, Rahman MM, Boldbaatar D, et al. The identification and characterization of lysozyme from the hard tick Haemaphysalis longicornis. Ticks Tick Borne Dis. 2010;1:178-85.

94. Buresova V, Hajdusek O, Franta Z, Sojka D, Kopacek P. IrAM-An alpha2macroglobulin from the hard tick Ixodes ricinus: characterization and function in phagocytosis of a potential pathogen Chryseobacterium indologenes. Dev Comp Immunol. 2009;33:489-98.

95. Chang HJ, Dhanasingh I, Gou X, Rice AM, Dushay MS. Loss of hemolectin reduces the survival of Drosophila larvae after wounding. Dev Comp Immunol. 2012;36:274-8.

96. Scherfer C, Karlsson C, Loseva O, Bidla G, Goto A, Havemann J, et al. Isolation and characterization of hemolymph clotting factors in Drosophila melanogaster by a pullout method. Curr Biol. 2004;14:625-9.

97. Lesch C, Goto A, Lindgren M, Bidla G, Dushay MS, Theopold U. A role for hemolectin in coagulation and immunity in Drosophila melanogaster. Dev Comp Immunol. 2007;31:1255-63.

98. Graca-Souza AV, Maya-Monteiro C, Paiva-Silva GO, Braz GR, Paes MC, Sorgine $\mathrm{MH}$, et al. Adaptations against heme toxicity in blood-feeding arthropods. Insect Biochem Mol Biol. 2006;36:322-35.

99. Ketterman AJ, Saisawang C, Wongsantichon J. Insect glutathione transferases. Drug Metab Rev. 2011;43:253-65.

100. Yamamoto K, Teshiba S, Shigeoka Y, Aso Y, Banno Y, Fujiki T, et al. Characterization of an omega-class glutathione S-transferase in the stress response of the silkmoth. Insect Mol Biol. 2011;20:379-86.
101. Balakrishnan B, Su S, Wang K, Tian R, Chen M. Identification, expression, and regulation of an omega class glutathione S-transferase in Rhopalosiphum padi (L.) (Hemiptera: Aphididae) under insecticide stress. Front Physiol. 2018;9:427.

102. Busby AT, Ayllón N, Kocan KM, Blouin EF, de la Fuente G, Galindo RC, et al. Expression of heat shock proteins and subolesin affects stress responses, Anaplasma phagocytophilum infection and questing behaviour in the tick, Ixodes scapularis. Med Vet Entomol. 2012;26:92-102.

103. Shahein YE, El-Rahim MT, Hussein NA, Hamed RR, El-Hakim AE, Barakat MM. Molecular cloning of a small heat shock protein (sHSPII) from the cattle tick Rhipicephalus (Boophilus) annulatus salivary gland. Int J Biol Macromol. 2010;47:614-22.

104. Chen B, Feder ME, Kang L. Evolution of heat-shock protein expression underlying adaptive responses to environmental stress. Mol Ecol. 2018;27:3040-54.

105. Lindquist S. The heat-shock response. Annu Rev Biochem. 1986:55:1151-91.

106. Galay RL, Umemiya-Shirafuji R, Mochizuki M, Fujisaki K, Tanaka T. Iron metabolism in hard ticks (Acari: Ixodidae): the antidote to their toxic diet. Parasitol Int. 2015;64:182-9.

107. Hajdusek O, Sojka D, Kopacek P, Buresova V, Franta Z, Sauman I, et al. Knockdown of proteins involved in iron metabolism limits tick reproduction and development. Proc Natl Acad Sci USA. 2009;106:1033-8.

108. Cook JD, Skikne BS, Baynes RD. Serum transferrin receptor. Annu Rev Med. 1993:44:63-74.

109. Scherz-Shouval R, Shvets E, Fass E, Shorer H, Gil L, Elazar Z. Reactive oxygen species are essential for autophagy and specifically regulate the activity of Atg4. EMBO J. 2007;26:1749-60.

\section{Publisher's Note}

Springer Nature remains neutral with regard to jurisdictional claims in published maps and institutional affiliations.
Ready to submit your research? Choose BMC and benefit from:

- fast, convenient online submission

- thorough peer review by experienced researchers in your field

- rapid publication on acceptance

- support for research data, including large and complex data types

- gold Open Access which fosters wider collaboration and increased citations

- maximum visibility for your research: over 100M website views per year

At BMC, research is always in progress.

Learn more biomedcentral.com/submissions 